Pontifícia Universidade C Católica

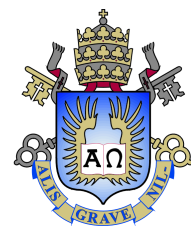

Luis Antonio Gómez Ardila

Deep Morin Singularities of the

McKean-Scovel Operator

Tese de Doutorado

Thesis presented to the Programa de Pós-graduação em Matemática, do Departamento de Matemática da PUC-Rio in partial fulfillment of the requirements for the degree of Doutor em Matemática.

Advisor: Prof. Carlos Tomei 


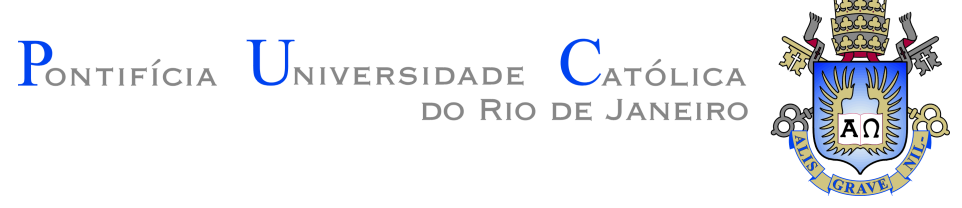

Luis Antonio Gómez Ardila

\section{Deep Morin Singularities of the McKean-Scovel Operator}

Thesis presented to the Programa de Pós-graduação em Matemática da PUC-Rio in partial fulfillment of the requirements for the degree of Doutor em Matemática. Approved by the Examination Committee:

Prof. Carlos Tomei

Advisor

Departamento de Matemática - PUC-Rio

Prof. Bernhard Ruf

Unimi

Prof. Nicolau Corção Saldanha

Departamento de Matemática - PUC-Rio

Prof. Ederson Moreira dos Santos

USP-São Carlos

Prof. Nivaldo de Góes Grulha Júnior USP-São Carlos

Prof. Sergey Galkin

Departamento de Matemática - PUC-Rio

Prof. João Marcos Bezerra do Ó

UFPB

Rio de Janeiro, September the 29th, 2021 
All rights reserved.

\section{Luis Antonio Gómez Ardila}

Electronic Engineer by Universidad Industrial de Santander (Bucaramanga, Colombia). Master in Mathematics by Universidad de los Andes (Bogotá, Colombia). Research interest: Operator Theory.

Bibliographic data

Gómez Ardila, Luis Antonio

Deep Morin Singularities of the McKean-Scovel Operator / Luis Antonio Gómez Ardila; advisor: Carlos Tomei. - 2021.

$54 \mathrm{f}$ : il. color. ; $30 \mathrm{~cm}$

Tese (doutorado) - Pontifícia Universidade Católica do Rio de Janeiro, Departamento de Matemática, 2021.

Inclui bibliografia

1. Matemática - Teses. 2. Análise - Teses. 3. Singularidades - Teses. 4. Operador. 5. Pontos Críticos. 6. Singularidades. 7. McKean-Scovel. 8. Morin. 9. Profundidade Arbitrária. I. Tomei, Carlos. II. Pontifícia Universidade Católica do Rio de Janeiro. Departamento de Matemática. III. Título. 


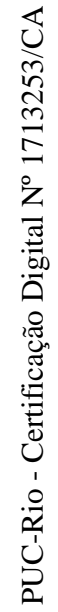

To V. 


\section{Acknowledgments}

This study was financed in part by the Coordenação de Aperfeiçoamento de Pessoal de Nível Superior - Brasil (CAPES) - Finance code 001. Also by the Fundação de Amparo à Pesquisa do Estado do Rio de Janeiro - FAPERJ.

Also, I am very grateful to...

Prof. PhD. Carlos Tomei, my teacher and adviser, by share with me the problem that this thesis is about. Also by his wonderful talks about Spectral Theory.

The Department of Mathematics of PUC-Rio, by giving me the opportunity to do my doctoral studies.

Profs. Ricardo Monturiol, Edilberto Reyes, Rafael Isaacs, Rafael Castro, Gildardo Guzmán and Sonia Sabogal at Universidad Industrial de Santander (Bucaramanga, Colombia).

Prof. Monika Winklmeier at Universidad de los Andes (Bogotá, Colombia). Yunelsy, by her great help during 2017-2. Thanks! 


\section{Abstract}

Gómez Ardila, Luis Antonio; Tomei, Carlos (Advisor). Deep Morin Singularities of the McKean-Scovel Operator. Rio de Janeiro, 2021. 54p. Tese de Doutorado - Departamento de Matemática, Pontifícia Universidade Católica do Rio de Janeiro.

The McKean-Scovel operator is the simplest nonlinear Sturm-Liouville operator acting on functions satisfying Dirichlet boundary conditions: its nonlinearity is just taking the square of the incoming function. This text contains a proof of a conjecture from the late '80: its critical set consists only of Morin singularities, which attain arbitrary depth.

\section{Keywords}

Operator; Critical Points; Singularities; McKean-Scovel; Morin; Arbitrary Depth. 


\section{Resumo}

Gómez Ardila, Luis Antonio; Tomei, Carlos. Singularidades de Morin Profundas do Operador McKean-Scovel. Rio de Janeiro, 2021. 54p. Tese de Doutorado - Departamento de Matemática, Pontifícia Universidade Católica do Rio de Janeiro.

O operador de McKean-Scovel agindo sobre funções que satisfazem condições de Dirichlet é o operador não-linear de Sturm-Liouville mais simples: a não-linearidade é elevar ao quadrado. Nesse texto, demonstra-se uma conjetura que de mais de trinta anos: seu conjunto crítico só contém singularidades de Morin, que podem ter profundidade arbitrária.

\section{Palavras-chave}

Operador; Pontos Críticos; Singularidades; McKean-Scovel; Morin; Profundidade Arbitrária. 


\section{Table of contents}

$\begin{array}{lll}0 & \text { Basic Notation } & 10\end{array}$

1 Introduction $\quad 12$

2 Morin Singularities $\quad 15$

$\begin{array}{lll}2.1 & \text { Spectral Properties of DF } & 15\end{array}$

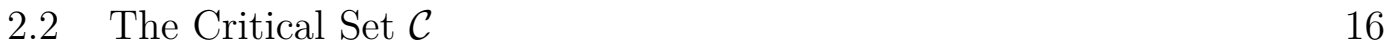

2.3 Morin Singularities of $F \quad 16$

2.3.1 The Thom-Boardman Stratification of the Critical Set 16

2.3.2 Morin Singularities as Level Sets 17

2.3.3 A Characterization of Functions in $\mathcal{Z}\left(M_{k}\right) \quad 20$

$3 \quad$ Every Singularity of $F$ is of Morin type 26

3.1 Computing grad $\widetilde{I}_{j}(u)$ on $\mathcal{Z}\left(M_{k}\right)$ for $j \leq k$

3.2 Zero is a Regular Value of $M_{k}$

3.3 Every critical point belongs to some $\mathcal{Z}\left(M_{k}\right) \backslash \mathcal{Z}\left(M_{k+1}\right)$

4 Constructing Singularities of Arbitrary Depth 35

$4.1 \quad$ Extending $M_{k} \quad 35$

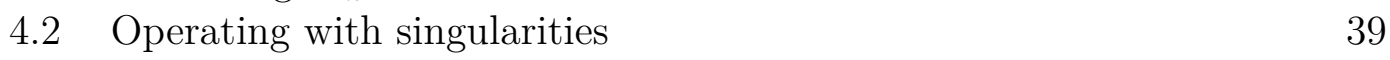

$\begin{array}{lll}4.2 .1 & \text { Scaling } & 40\end{array}$

4.2.2 Juxtaposition 41

4.2.3 Symmetrization: from odd depth $k$ to depth $k+1$

4.3 Some deep singularities in $X^{\delta} \quad 43$

4.4 Adjustable Functions 44

4.4.1 From even $k$ to $k+1 \quad 45$

4.4.2 Existence of Adjustable Functions 48

4.5 There exist Morin singularities of arbitrary depth 50

$\begin{array}{lll}\text { 4.6 An application of Theorem 1.0.2 } & 51\end{array}$

Bibliography $\quad 52$ 
Functions, just like living beings, are characterized by their singularities. 


\section{0}

\section{Basic Notation}

- $\mathbb{N}, \mathbb{N}_{0}$ and $\mathbb{R}$ are the positive integers, non-negative integers and real numbers, respectively.

- $\|\cdot\|_{\text {sup }}$ denotes the supremum norm (or uniform norm) on $C([a, b]$ ).

- Norm and inner product on $L^{2}([a, b])$ are denoted by $\|\cdot\|$ and $\langle\cdot, \cdot\rangle$.

- If $V$ is an inner product space, $\langle\cdot, \cdot\rangle_{V}$ denotes its inner product and $\|\cdot\|_{V}$ the induced norm.

- A function $w:[a, b] \rightarrow \mathbb{R}$ is Dirichlet if it satisfies Dirichlet boundary conditions, $w(a)=w(b)=0$.

- The function $u:[a, b] \rightarrow \mathbb{R}$ is absolutely continuous if and only if there exists $v \in L^{1}([a, b])$ such that

$$
u(x)=u(a)+\int_{a}^{x} v(t) d t, \quad x \in[a, b] .
$$

The function $v$ is denoted by $u^{\prime}$.

- $A C([a, b])=\{u:[a, b] \rightarrow \mathbb{R} \mid u$ is absolutely continuous $\}$.

- $H^{1}([a, b])=\left\{u \in A C([a, b]) \mid u^{\prime} \in L^{2}([a, b])\right\}$.

- $H_{0}^{1}([a, b])=\left\{u \in H^{1}([a, b]) \mid u(a)=u(b)=0\right\}$ is a Hilbert space with the inner product $\langle u, v\rangle_{H_{0}^{1}}:=\left\langle u^{\prime}, v^{\prime}\right\rangle_{L^{2}}$. The norm $\|\cdot\|_{H_{0}^{1}}$ induced by $\langle u, v\rangle_{H_{0}^{1}}$ is equivalent to $\|\cdot\|_{H^{1}}$ on $H_{0}^{1}([a, b])$.

- $H_{D}^{2}([a, b])=H^{2}([a, b]) \cap H_{0}^{1}([a, b])=\left\{u \in H_{0}^{1}([a, b]) \mid u^{\prime} \in H^{1}([a, b])\right\}$.

- For real Banach spaces $E_{1}, E_{2}$,

$$
\mathcal{B}\left(E_{1}, E_{2}\right)=\left\{T: E_{1} \rightarrow E_{2} \mid T \text { is linear continuous }\right\}
$$

with the usual operator norm, $\|T\|=\sup _{\|v\|=1}\|T v\|$.

- For a differentiable map $G: E_{1} \rightarrow E_{2}$, the derivative (Jacobian) of $G$ at the point $u \in E_{1}$ is denoted by $D G(u) \in \mathcal{B}\left(E_{1}, E_{2}\right)$. For every nonzero $v \in E_{1}$, the directional derivative of $G$ at $u \in E_{1}$ along $v$ is $\partial_{v} G(u)=D G(u) \cdot v$. 
- For a real Hilbert space $H$ and a differentiable functional $\varphi: H \rightarrow \mathbb{R}$, the $\operatorname{gradient} \operatorname{grad} \varphi(u) \in H$ is the only element of $H$ such that $D \varphi(u) \cdot v=$ $\langle\operatorname{grad} \varphi(u), v\rangle_{H}$ for every $v \in H$.

- For a real valued map $f, \mathcal{Z}(f)$ denotes the zero set of $f$,

$$
\mathcal{Z}(f)=\{u \in \operatorname{Dom}(f) \mid f(u)=0\}
$$

- For $M$ a smooth manifold and $p \in M, T_{p} M$ stands for the tangent space of $M$ at the point $p$.

- The prime ' stands for the derivative in the real variable $x\left(\right.$ i.e., ${ }^{\prime}=\frac{d}{d x}$ ).

- A function $f$ is an $o\left(x^{n}\right)$-function, $f=o\left(x^{n}\right)$, as $x \rightarrow 0$ if and only if

$$
\lim _{x \rightarrow 0}\left|\frac{f(x)}{x^{n}}\right|=0
$$

- For $C^{1}$ functions $f, g:[a, b] \rightarrow \mathbb{R}$, the Wronskian of $f$ and $g$ is the function $[f, g]:[a, b] \rightarrow \mathbb{R}$ defined by $[f, g](x)=f(x) g^{\prime}(x)-f^{\prime}(x) g(x)$. 


\section{Introduction}

The Ambrosetti-Prodi theorem [1] has been the starting point of active research for decades. After contributions by Manes-Micheletti [2] and BergerPodolak [3], it may be stated as follows. For $\Omega \subset \mathbb{R}^{n}$, a bounded domain with smooth boundary, consider the Dirichlet Laplacian

$$
-\Delta: X=H^{2}(\Omega) \cap H_{0}^{1}(\Omega) \rightarrow Y=L^{2}(\Omega)
$$

with eigenvalues

$$
\lambda_{1}<\lambda_{2} \leq \lambda_{3} \leq \cdots
$$

Given a smooth, strictly convex function $f: \mathbb{R} \rightarrow \mathbb{R}$ satisfying

$$
a=\lim _{x \rightarrow-\infty} f^{\prime}(x)<\lambda_{1}<\lim _{x \rightarrow+\infty} f^{\prime}(x)=b<\lambda_{2}
$$

define

$$
F: X \rightarrow Y, \quad u \mapsto-\Delta u+f(u)
$$

Theorem 1.0.1 The map $F$ is a global fold. More explicitly, there are global homeomorphisms defined on $X$ and $Y$ which convert $F$ to the map

$$
\begin{aligned}
G: \quad Z \times \mathbb{R} & \rightarrow Z \times \mathbb{R} \\
(z, t) & \mapsto\left(z, t^{2}\right),
\end{aligned}
$$

for some real Banach space $Z$.

It is a wonderful fact that a nonlinear differential operator may admit such a simple description of its global geometry, possibly the simplest if we exclude homeomorphisms. The different arguments leading to the result emphasized the importance of local theory: the map is not a local homemomorphism at each point of its domain. But such critical points turned out to be the simplest possible deviations from the inverse function theorem: within the classification from singularity theory, they are folds.

The search for additional contexts led to further examples in which global geometry may still be described in simple terms (Church-Timourian folds [4]) 
and others in which different singularities were identified (Church-Timourian cusps $[4,5,14,15])$.

One interesting case was suggested by McKean and Scovel [6]. Set $X=H_{D}^{2}([0,1])$ and $Y=L^{2}([0,1])$. The McKean-Scovel operator $F: X \rightarrow Y$ is

$$
F(u)=-u^{\prime \prime}+\frac{1}{2} u^{2}
$$

Let $\mathcal{C}$ denotes the critical set of $F$,

$$
\mathcal{C}=\{u \in X \mid D F(u) \in \mathcal{B}(X, Y) \text { is non-invertible }\}
$$

In opposition to the situation in the Ambrosetti-Prodi theorem, the nonlinearity $f(x)=\frac{1}{2} x^{2}$ interacts with all eigenvalues of the linear term. The onedimensional context ensures that critical points (i.e., points in $\mathcal{C}$ ) are potentially simple: the dimension of the kernel of the derivative $D F(u): X \rightarrow Y$ is never larger than one. With an additional requirement of transversality described in detail in Section 2.3, such critical points are called Morin singularities $([17,21])$.

Morin singularities are classified by their depth: after local changes of variable (see $[9,14]$ for the infinite-dimensional case), the function $F$ near a singularity of depth $k$ takes the form

$$
\begin{aligned}
& \left(z, s_{1}, \ldots, s_{k-1}, t\right) \in Z \times \mathbb{R}^{k} \mapsto \\
& \quad\left(z, s_{1}, \ldots, s_{k-1}, t^{k+1}+s_{1} t^{k-1}+s_{2} t^{k-2}+\cdots+s_{k-1} t\right) \in Z \times \mathbb{R}^{k} .
\end{aligned}
$$

In [7] McKean presented a proof that $F$ has Morin singularities of arbitrary depth. Unfortunately, Ruf identified an error [8], and the original claim became a conjecture. In this thesis we vindicate it.

Theorem 1.0.2 A. Every critical point of $F$ is a Morin singularity.

$B$. Any dense subspace $D \subset X$ contains singularities of arbitrary depth.

Deeper singularities did not receive as much attention as folds and cusps, the first two cases in the hierarchy of Morin singularities. Theoretical aspects - specifically, the counterpart of the usual characterization for infinite dimensional spaces - have been clarified in [9] and [14]. Conditions yielding existence of arbitrarily deep singularities for operators $F(u)=-u^{\prime}+f(u)$ acting on periodic functions were obtained in [14], solving a conjecture of Cafagna-Donati [10].

The first step in the proof of Theorem 1.0.2 is identifying Morin singularities of depth $k$ in a concrete fashion. As we shall see in Sections 2.3 and 3.2, 
there are real smooth maps $M_{i}: X \rightarrow \mathbb{R}^{i}, i=1,2, \ldots$, for which $u \in X$ is a Morin singularity of depth $k$ if and only if

(i) $M_{k}(u)=0, M_{k+1}(u) \neq 0$,

(ii) Zero is a regular value of $M_{k}$, i.e., $D M_{k}(u): X \rightarrow \mathbb{R}^{k}$ is surjective.

In Section 2.3.2 we prove that for each critical point $u$ there is $j \in \mathbb{N}$ such that $M_{j}(u) \neq 0$. Thus every singularity of $F$ is indeed of Morin type.

In Chapter 4 , we show that every $M_{k}$ has a root in $X$. The maps $M_{k}$ admit real smooth extensions $\hat{M}_{k}: H^{-1}([0,1]) \rightarrow \mathbb{R}^{k}$ (Section 4.1$)$. We are then led to consider solutions defined by finite sums of deltas $\sum_{j} c_{j} \delta_{x_{j}}, x_{j} \in(0,1)$. In Proposition 2.3.4 we present juxtaposition: from two functions in adjacent intervals which are zeros of the $\hat{M}_{k}$ 's, we juxtapose domains and rescale the resulting interval back to $[0,1]$ so as to obtain not only another zero of $\hat{M}_{k}$, but a curve of zeros by inserting a $\delta$ of arbitrary weight at the juncture of both domains. Iteration of this process leads to zeros of $\hat{M}_{k}$ with additional degrees of freedom. The difficulty now lies in identifying appropriate weights to such degrees of freedom to obtain a root of $\hat{M}_{k+1}$. Odd values $k$ are easier to handle (Proposition 4.2.3). When $k$ is even, there are specific locations $x_{s} \in(0,1)$ - the points of adjustment of Section 4.4 - for which the insertion of a weighted $\delta_{x_{s}}$ suffices to obtain a root $u_{*}$ of $\hat{M}_{2 k+1}$. A transversality argument then implies that $u_{*}$ may be perturbed so as to obtain a root of $M_{2 k+1}$ on arbitrary dense subspaces of $X$, and those are indeed Morin singularities of depth larger than $k$ of $F$. A substantial amount of symbolic computation led us to the construction of deep singularities. Some examples are shown in Section 4.3.

Propositions 2.3.5 and 3.1.1 are of a strong algebraic nature. Extending the result for additional nonlinearities would require a different approach. 


\section{2}

\section{Morin Singularities}

For $X=H_{D}^{2}([0,1])$ and $Y=L^{2}([0,1])$, the McKean-Scovel operator is

$$
F: X \rightarrow Y, \quad F(u)=-u^{\prime \prime}+\frac{1}{2} u^{2} .
$$

Morin singularities of the McKean-Scovel operator are described in terms of eigenvalues and eigenvectors of its Jacobians $D F(u)$.

In Section 2.1 we encapsulate some standard spectral properties of $D F(u)$ (see $[6,20,22,23])$. In Section 2.2, we introduce the critical set $\mathcal{C}$ of $F$. Deeper singularities are defined in Section 2.3.

\section{1}

\section{Spectral Properties of $D F$}

Let $M_{u}: X \rightarrow Y$ be the operator of multiplication by $u$.

Proposition 2.1.1 The map $F: X \rightarrow Y$ is proper, (real) analytic and its derivative at each $u \in X$ is the bounded operator

$$
D F(u): X \rightarrow Y, \quad D F(u)=-\frac{d^{2}}{d x^{2}}+M_{u}
$$

$D F(u): X \subset Y \rightarrow Y$ is a self-adjoint operator and its spectrum $\sigma(D F(u))$ consists of simple eigenvalues converging to infinity,

$$
\lambda_{1}(u)<\lambda_{2}(u)<\cdots<\lambda_{n}(u)<\cdots \rightarrow+\infty .
$$

Let $\phi_{n}(u)$ be the eigenfunction associated with $\lambda_{n}(u)$, normalized so as $\left\|\phi_{n}(u)\right\|=1$ (in the $L^{2}$-norm) and $\phi_{n}(u)^{\prime}(0)>0$. It has $n-1$ simple zeros in the open interval $(0,1)$. The operator $D F(u): X \rightarrow Y$ is Fredholm of index zero, with $\operatorname{dim} \operatorname{ker}\left(D F(u)-\lambda_{n}(u)\right)=1$. The eigenmaps $u \in X \mapsto \lambda_{n}(u) \in \mathbb{R}$ and $u \in X \mapsto \phi_{n}(u) \in X$ are smooth and, for each $u, v \in X, v \neq 0$,

$$
\begin{gathered}
\partial_{v} \lambda_{n}(u)=\left\langle\phi_{n}(u)^{2}, v\right\rangle, \\
\left(D F(u)-\lambda_{n}(u)\right) \cdot \partial_{v} \phi_{n}(u)=-v \phi_{n}(u)+\left[\partial_{v} \lambda_{n}(u)\right] \phi_{n}(u) .
\end{gathered}
$$

On lines of $X$, the eigenvalue maps restrict to real analytic maps. 


\section{2}

\section{The Critical Set $\mathcal{C}$}

Let $\mathcal{C}$ denote the critical set of $F$,

$$
\begin{aligned}
\mathcal{C} & =\{u \in X \mid D F(u) \in \mathcal{B}(X, Y) \text { is non-invertible }\} \\
& =\{u \in X \mid \operatorname{dim} \operatorname{ker} D F(u)=1\} \\
& =\left\{u \in X \mid \exists \varphi \in X \backslash\{0\},-\varphi^{\prime \prime}+u \varphi=0\right\} .
\end{aligned}
$$

Define the zero set $\mathcal{C}_{n}=\left\{u \in X \mid \lambda_{n}(u)=0\right\}$ of the eigenmap $\lambda_{n}$. The following result is a small variation of a theorem in [6].

Proposition 2.2.1 Each $\mathcal{C}_{n}$ is a smooth submanifold of $X$ of codimension one. The sets are disjoint and isolated from each other. The critical set $\mathcal{C}=\cup_{n \in \mathbb{N}} \mathcal{C}_{n}$ also is a smooth submanifold of $X$ of codimension one.

Proof. The set $\mathcal{C}_{n}$ is nonempty and connected - as is shown in Theorem $\mathrm{C}$ [13], it is a graph over the orthogonal complement of $\sin (\pi x)$. For $u \in \mathcal{C}_{n}$, from Proposition 2.1.1, the eigenfunction $\phi_{n}(u)$ has $n+1$ (simple) zeros in $[0,1]$ : the sets $\mathcal{C}_{n}$ are disjoint. As $\phi_{n}(u) \neq 0$, from equation (2.1) we have that $D \lambda_{n}(u)$ is nonzero and therefore, by the Implicit Function Theorem, $\mathcal{C}_{n}$ is a smooth submanifold of $X$ of codimension one. In particular, close to $u \in \mathcal{C}_{n}$, one cannot have $\lambda_{m}(u)=0$ for $m \neq n$ : the sets are isolated, so that $\mathcal{C}$ is also a manifold.

\section{3}

\section{Morin Singularities of $F$}

We recall the definition of a Morin singularity of depth $k$. The geometric definition yields a set of equations characterizing such singularities for the map $F$.

\subsection{1}

\section{The Thom-Boardman Stratification of the Critical Set}

Given a smooth map $F: X \rightarrow Y$, we stratify its critical set according to the Thom-Boardman symbols [21]. Here, $X$ and $Y$ are real Banach spaces, and we suppose that Jacobians $D F(u): X \rightarrow Y$ are Fredholm operators of index zero. Define the Thom set $\Sigma^{J}$ for a multi-index $J$ with entries in $\mathbb{N}_{0}$. For $i \in \mathbb{N}_{0}$,

$$
\Sigma^{i}=\Sigma^{i}(F)=\{u \in X \mid \operatorname{dim} \operatorname{ker} D F(u)=i\} .
$$


For $i_{1}, i_{2} \in \mathbb{N}_{0}$ with $i_{1} \geq i_{2}$, if $\Sigma^{i_{1}}$ is a manifold, set

$$
\Sigma^{i_{1}, i_{2}}=\Sigma^{i_{2}}\left(F \uparrow_{\Sigma^{i_{1}}}\right)
$$

Inductively, suppose $J=\left(i_{1}, \ldots, i_{k}\right)$ is a non-increasing sequence in $\mathbb{N}_{0}$ and $\Sigma^{J}$ is a manifold. Then, for $i_{k+1} \in \mathbb{N}_{0}$ with $i_{k} \geq i_{k+1}$,

$$
\Sigma^{i_{1}, \ldots, i_{k}, i_{k+1}}=\Sigma^{i_{1}, \ldots, i_{k}, i_{k+1}}(F)=\Sigma^{i_{k+1}}\left(F \uparrow_{\Sigma^{J}}\right) .
$$

Clearly, if $\Sigma^{i_{1}, \ldots, i_{k}}$ is a manifold and $i_{k} \geq i_{k+1}$ then

$$
\sum^{i_{1}, \ldots, i_{k}} \supseteq \sum^{i_{1}, \ldots, i_{k}, i_{k+1}} .
$$

We are interested in very special multi-indices: for the McKean-Scovel operator $F$, we have $\operatorname{dim} \operatorname{ker} D F(u) \leq 1$. Since $\Sigma^{1}=\mathcal{C}$ is a manifold, $\Sigma^{1,1}$ makes sense.

Set $[k]=(1, \ldots, 1) \in \mathbb{R}^{k}$. For $k \geq 1$, if $\Sigma^{[k]}$ is a manifold, the set of Morin singularities of depth $k$ is

$$
\mathcal{S}_{k}=\Sigma^{[k]} \backslash \Sigma^{[k+1]} .
$$

The set of all Morin singularities is $\mathcal{S}=\bigcup_{k \in \mathbb{N}} \mathcal{S}_{k}$. Clearly $\mathcal{S} \subseteq \mathcal{C}$.

In the notation above, Theorem 1.0.2 becomes

Theorem 2.3.1 (A) Every critical point of $F$ is a Morin singularity, $\mathcal{C}=\mathcal{S}$.

(B) There are singularities of arbitrary depth: $\mathcal{S}_{k} \neq \emptyset$ for every $k \in \mathbb{N}$.

\subsection{2}

\section{Morin Singularities as Level Sets}

Define $\Sigma^{[0]}=X$. The Thom-Boardman stratification may be described as the zero-levels of a set of functionals: $\Sigma^{[k]}=\mathcal{Z}\left(I_{k}\right)$, the zero set of appropriate $I_{k}: \Sigma^{[k-1]} \rightarrow \mathbb{R}$

To verify if $u_{*} \in X$ is a Morin singularity of the map $F$, we first check if $u_{*}$ is critical, i.e, if $u_{*}$ is a zero of the eigenvalue map $u \mapsto \lambda_{n}(u)$ for some $n \in \mathbb{N}$. Set $\lambda=\lambda_{n}$ and $\phi=\phi_{n}$. If $\Sigma^{[1]}=\mathcal{C}$ is a manifold (which is the case for the map $F$ ), we proceed to verify if $u_{*} \in \Sigma^{[2]}$, i.e., if $\phi\left(u_{*}\right)$ lies in $T_{u_{*}} \mathcal{C}$, i.e., if $u_{*}$ is a zero of the map $u \mapsto \partial_{\phi(u)} \lambda(u)$. Iteration of this argument suggests the definitions

$$
I_{1}: \Sigma^{[0]} \rightarrow \mathbb{R}, \quad u \mapsto I_{1}(u)=\lambda(u),
$$

and, for $k \geq 1$, if $\Sigma^{[k]}$ is a manifold, 


$$
I_{k+1}: \Sigma^{[k]} \rightarrow \mathbb{R}, \quad u \mapsto I_{k+1}(u)=\partial_{\phi(u)} I_{k}(u)=\partial_{\phi(u)}^{(k)} \lambda(u) .
$$

The above definition makes sense: $u \in \Sigma^{[k]}$ implies that $\phi(u)$ belongs to $T_{u} \Sigma^{[k-1]}$ and so directional derivatives of the restrictions $I_{k}$ are well defined.

Proposition 2.3.2 For $k \geq 0$, if $\Sigma^{[k]}$ is a manifold then

$$
\Sigma^{[k+1]}=\mathcal{Z}\left(I_{k+1}\right)=\left\{u \in X \mid \partial_{\phi(u)}^{(0)} \lambda(u)=\cdots=\partial_{\phi(u)}^{(k)} \lambda(u)=0\right\}
$$

Proof. The statement is true for $k=0$ since $\Sigma^{[1]}=\{u \in X \mid \lambda(u)=0\}=\mathcal{Z}\left(I_{1}\right)$. Assume it is true for some $k \geq 0$ and suppose $\Sigma^{[k]}$ is a manifold. Then

$$
\begin{aligned}
\Sigma^{[k+1]} & =\left\{u \in \Sigma^{[k]} \mid \phi(u) \in T_{u} \Sigma^{[k]}\right\}=\left\{u \in \Sigma^{[k]} \mid \phi(u) \in T_{u} \mathcal{Z}\left(I_{k}\right)\right\} \\
& =\left\{u \in \Sigma^{[k]} \mid D I_{k}(u) \cdot \phi(u)=0\right\}=\left\{u \in \Sigma^{[k]} \mid \partial_{\phi(u)} I_{k}(u)=0\right\} \\
& =\left\{u \in \Sigma^{[k]} \mid I_{k+1}(u)=0\right\}=\mathcal{Z}\left(I_{k+1}\right) .
\end{aligned}
$$

At some point, we must prove that the sets $\Sigma^{[k]}$ are indeed (nested) manifolds. A simple modification of the above construction gives a geometric perspective. Since the maps $I_{1}=\lambda: X \rightarrow \mathbb{R}$ and $\phi: X \rightarrow X$ are smooth, globally defined, the functionals $I_{k}: \Sigma^{[k-1]} \rightarrow \mathbb{R}$ admit an obvious extension $\widetilde{I}_{k}: X \rightarrow \mathbb{R}$

$$
\widetilde{I}_{1}=I_{1}, \quad \widetilde{I}_{k+1}(u)=\partial_{\phi(u)}^{(k)} \lambda(u)=\partial_{\phi(u)} \widetilde{I}_{k}(u)
$$

Set

$$
M_{k}: X \rightarrow \mathbb{R}^{k}, \quad u \mapsto\left(\widetilde{I}_{1}(u), \ldots, \widetilde{I}_{k}(u)\right),
$$

and $\mathcal{Z}\left(M_{k}\right)=M_{k}^{-1}(0)$. If zero is a regular value of $M_{k}$, then $\mathcal{Z}\left(M_{k}\right)$ is a submanifold of $X$ of codimension $k$, and then clearly $\mathcal{Z}\left(M_{k}\right)=\Sigma^{[k]}$. We already know that $\mathcal{Z}\left(M_{1}\right)=\mathcal{C}_{n}$. Clearly

$$
\mathcal{Z}\left(M_{1}\right) \supset \mathcal{Z}\left(M_{2}\right) \supset \mathcal{Z}\left(M_{3}\right) \supset \cdots
$$

Theorem 1.0.2 in this notation corresponds to the following statements.

Theorem 2.3.3 For each $u \in X$ :

i) Zero is a regular value of $M_{k}$.

ii) $u \in \mathcal{S}_{k}$ if and only if $M_{k}(u)=0$ and $M_{k+1}(u) \neq 0$.

iii) If $u \in \mathcal{C}$, there exists $j \in \mathbb{N}$ such that $M_{j}(u) \neq 0$.

iv) For each $k \in \mathbb{N}, \mathcal{Z}\left(M_{k}\right) \neq \emptyset$. 
From (i), the sets $\mathcal{Z}\left(M_{k}\right)$ are manifolds of codimension $k$ and $\mathcal{Z}\left(M_{k}\right)=$ $\Sigma^{[k]}$. Item (ii) characterizes Morin singularities of depth $k$ in terms of $M_{k}$ and $M_{k+1}$. Items (iii) and (iv) are respectively parts (A) and (B) of Theorem 1.0.2.

To obtain a more concrete description of the maps $M_{k}$, take successive directional derivatives along $\phi(u)$ of the eigenvalue equation

$$
D F(u) \cdot \phi(u)=-\phi(u)^{\prime \prime}+u \phi(u)=\lambda(u) \phi(u) .
$$

To simplify notation define the maps $z_{k}: X \rightarrow X$,

$$
z_{1}(u)=\phi(u), \quad z_{k+1}(u)=\partial_{\phi(u)}^{(k)} z_{1}(u)=\partial_{\phi(u)} z_{k}(u)
$$

together with the functions $r_{k}: X \rightarrow X$,

$$
r_{1} \equiv 0, \quad r_{k+1}(u)=z_{1}(u) z_{k}(u)+\partial_{\phi(u)} r_{k}(u)
$$

The functions $z_{j}(u)$ are Dirichlet.

Proposition 2.3.4 For each $u \in X$, and all $j \in \mathbb{N}$,

$$
-z_{j}(u)^{\prime \prime}+u z_{j}(u)=-r_{j}(u)+\sum_{i=0}^{j-1}\left(\begin{array}{c}
j-1 \\
i
\end{array}\right) \widetilde{I}_{i+1}(u) z_{j-i}(u),
$$

where

$$
r_{j+1}(u)=\sum_{i=1}^{j}\left(\begin{array}{l}
j \\
i
\end{array}\right) z_{i}(u) z_{j+1-i}(u) .
$$

If $u \in \mathcal{Z}\left(M_{k}\right)$ then

$$
\begin{cases}-z_{j}(u)^{\prime \prime}+u z_{j}(u) & =-r_{j}(u), \quad j=1, \ldots, k \\ -z_{k+1}(u)^{\prime \prime}+u z_{k+1}(u) & =-r_{k+1}(u)+\tilde{I}_{k+1}(u) \phi(u) .\end{cases}
$$

Moreover,

$$
\tilde{I}_{k+1}(u)=\left\langle r_{k+1}(u), \phi(u)\right\rangle .
$$

Proof. The formula for $r_{p}$ is clear for $p=2$. We drop the obvious dependence on $u$ and verify the formula for $r_{p+1}$ from $r_{p}$ :

$$
\begin{aligned}
r_{p+1} & =z_{1} z_{p}+\partial_{\phi} r_{p}=z_{1} z_{p}+\partial_{\phi}\left(\sum_{i=1}^{p-1}\left(\begin{array}{c}
p-1 \\
i
\end{array}\right) z_{i} z_{p-i}\right) \\
& =z_{1} z_{p}+\sum_{i=1}^{p-1}\left(\begin{array}{c}
p-1 \\
i
\end{array}\right) z_{i+1} z_{p-i}+\sum_{i=1}^{p-1}\left(\begin{array}{c}
p-1 \\
i
\end{array}\right) z_{i} z_{p+1-i} \\
& =z_{1} z_{p}+\sum_{i=2}^{p}\left(\begin{array}{c}
p-1 \\
i-1
\end{array}\right) z_{i} z_{p+1-i}+\sum_{i=1}^{p-1}\left(\begin{array}{c}
p-1 \\
i
\end{array}\right) z_{i} z_{p+1-i} \\
& =z_{1} z_{p}+\sum_{i=2}^{p-1}\left[\left(\begin{array}{c}
p-1 \\
i-1
\end{array}\right)+\left(\begin{array}{c}
p-1 \\
i
\end{array}\right)\right] z_{i} z_{p+1-i}+p z_{1} z_{p} \\
& =z_{1} z_{p}+\sum_{i=2}^{p-1}\left(\begin{array}{c}
p \\
i
\end{array}\right) z_{i} z_{p+1-i}+p z_{1} z_{p}=\sum_{i=1}^{p}\left(\begin{array}{c}
p \\
i
\end{array}\right) z_{i} z_{p+1-i} .
\end{aligned}
$$


The differential equation is obtained by taking successive derivatives of the eigenvalue equation (2.3) at $u$ along $\phi(u)$.

For $u \in \mathcal{Z}\left(M_{k}\right)$, the system (2.6) is obtained from (2.4) making $\tilde{I}_{1}(u)=$ $\cdots=\tilde{I}_{k}(u)=0$. We now consider the expression for $\tilde{I}_{k+1}(u)$. Recall that $D F(u): X \subset Y \rightarrow Y$ is a self-adjoint, Fredholm operator of index 0 and ker $D F(u)$ is spanned by $z_{1}(u)=\phi(u)$. Solvability of

$$
D F(u) \cdot z_{k+1}(u)=-r_{k+1}(u)+\tilde{I}_{k+1}(u) \phi(u)
$$

is equivalent to $\left\langle-r_{k+1}(u)+\tilde{I}_{k+1}(u) \phi(u), \phi(u)\right\rangle=0$. As $\|\phi(u)\|=1$, equation (2.7) holds.

A brief summary of the constructions in this section is convenient. We take iterated directional derivatives along of the $L^{2}$-normalized eigenfunction $\phi(u)$ of two functions, the eigenvalue map $\lambda: X \rightarrow \mathbb{R}$ and the eigenfunction map $\phi: X \rightarrow X$, defining the maps $\widetilde{I}_{j}$ and $z_{j}$. From $z_{1}, \ldots, z_{j}$, we obtained $r_{j+1}$ and there are differential equations relating $\widetilde{I}_{j}, z_{j}$ and $r_{j}$ : the Dirichlet functions $z_{j}$ solve a system of equations. Uniqueness of solution definitely does not hold at a critical point $u$, as the equation for $j=1$ is solved by multiples of the eigenfunction $z_{1}(u)=\phi(u)$.

In the next section, we parameterize the set of solutions to this system in terms of an initial value problem for the same equations, leading to Proposition 2.3.8, a characterization of functions $u \in \mathcal{Z}\left(M_{k}\right)$ in terms of the existence of Dirichlet solutions for the system.

\subsection{3}

\section{A Characterization of Functions in $\mathcal{Z}\left(M_{k}\right)$}

Proposition 2.3.4 leads us to consider a system of differential equations, which we now study as an initial value problem. More precisely, we relate vector solutions $w$ and $\widetilde{w}$ of the (nonlinear) IVP

$$
\left\{\begin{array}{c}
-y_{j}^{\prime \prime}+u y_{j}=-q_{j}, \quad j=1, \ldots, k+1, \\
q_{1}=0, \quad q_{j+1}=\sum_{i=1}^{j}\left(\begin{array}{l}
j \\
i
\end{array}\right) y_{i} y_{j+1-i}, \quad j=1, \ldots, k, \\
y_{j}(0)=0, \quad j=1, \ldots, k+1,
\end{array}\right.
$$

with additional conditions on the derivative,

$$
y^{\prime}(0)=e_{1}, \quad \tilde{y}^{\prime}(0)=a \in \mathbb{R}^{k+1}, \quad a_{1} \neq 0 .
$$

Here $e_{1}, \ldots, e_{k+1}$ are the canonical vectors of $\mathbb{R}^{k+1}$. We write the system in matrix form. The expressions for $q_{j}$ are quadratic forms on $y, q_{j}=q_{j}(y)=$ $\left\langle Q_{j} y, y\right\rangle: Q_{1}=0$ and, for $j \geq 2$, the nonzero entries of the symmetric matrix 
$Q_{j}$ are

$$
\left(Q_{j}\right)_{s, j-s}=\frac{1}{2}\left[\left(\begin{array}{c}
j-1 \\
s
\end{array}\right)+\left(\begin{array}{c}
j-1 \\
j-s
\end{array}\right)\right] .
$$

The range of $Q_{j}$ is $\operatorname{span}\left\{e_{1}, \ldots, e_{j-1}\right\}$. Write $\left(v_{j}\right)_{j}$ for $v=\left(v_{1}, \ldots, v_{k+1}\right)$, so that $q=q(y)=\left(\left\langle Q_{j} y, y\right\rangle\right)_{j}$. In matrix notation, system $(2.8)$ is

$$
-y^{\prime \prime}+u y=-\left(\left\langle Q_{j} y, y\right\rangle\right)_{j}=-q(y), \quad y(0)=0 .
$$

Proposition 2.3.5 Let $w$ and $\widetilde{w}$ be the two solutions of the IVP (2.8). Then there is a constant lower triangular matrix $M$ for which

$$
\widetilde{w}=M w, \quad q(\widetilde{w})=M q(w),
$$

where

(a) for $j=1, \ldots, k+1, m_{j, j}=a_{1}^{j}, \quad$ (b) $M e_{1}=a$.

(c) for $j \geq 2$ and $s \in\{2, \ldots, j\}$, and any $t \in\{1, \ldots, s-1\}$,

$$
m_{j, s}=\frac{1}{\left(\begin{array}{c}
s-1 \\
t
\end{array}\right)} \sum_{i=t}^{j-s+t}\left(\begin{array}{c}
j-1 \\
i
\end{array}\right) m_{i, t} m_{j-i, s-t} .
$$

The proof requires preparation. The lemma below is strictly algebraic.

Lemma 2.3.6 Write $q=q(w)=\left(\left\langle Q_{j} w, w\right\rangle\right)_{j}$ for $Q_{j}$ as above. Then there is a lower triangular $(k+1) \times(k+1)$ matrix $M$ for which, for all $w \in \mathbb{R}^{k+1}$,

$$
M q(w)=q(M w)
$$

The matrix $M$ is uniquely determined by its first column, provided $m_{1,1} \neq 0$. Moreover, $m_{j, j}=a_{1}^{j} \neq 0$ and thus $M$ is invertible.

We say $M$ is an IVP matrix. Let $m_{i}=M e_{i}$ be the $i$-th column of $M$. Proof. For the bilinear extension $b(y, z)=\left(\left\langle Q_{j} y, z\right\rangle\right)_{j}$ of the quadratic form $q$,

$$
M b(y, z)=b(M y, M z), \quad y, z \in \mathbb{R}^{k+1},
$$

and, for arbitrary canonical vectors $y=e_{s}$ and $z=e_{r}$, we must then obtain

$$
M b\left(e_{s}, e_{r}\right)=b\left(m_{s}, m_{r}\right), \quad r, s=1, \ldots, k+1 .
$$

For $r=1$ and arbitrary $s$, the expressions become

$$
m_{1+s}=\frac{1}{\left(Q_{s+1}\right)_{1, s}} b\left(m_{s}, m_{1}\right) .
$$


Thus $m_{2}$ is obtained from (the given first column) $m_{1}$, and $m_{3}$ from $m_{2}$, successively up to $m_{k+1}$. These formulae define $M$ as a triangular matrix. Indeed, for some number $c, m_{1+s}=c b\left(m_{1}, m_{s}\right)=c b\left(m_{s}, m_{1}\right)$. For $j=1$, we have $Q_{j}=0$ and $\left(m_{2}\right)_{1}=0$. Now induce and use that only the first $j-1$ coordinates of $Q_{j} m_{1}$ are possibly nonzero. From equation (2.11), induction also yields $m_{j, j}=a_{1}^{j}$.

The rest of the proof consists of showing that the other restrictions, obtained by setting $r \neq 1$ and arbitrary $s$, are automatically satisfied. We must prove

$$
m_{r+s}=\frac{1}{\left(Q_{r+s}\right)_{r, s}} b\left(m_{s}, m_{r}\right), \quad r+s \leq k+1 .
$$

The restrictions follow from those we have chosen to define $M$ if we prove that, for $r=1, \ldots, k, s=2, \ldots, k+1$,

$$
\frac{1}{\left(Q_{r+s}\right)_{r, s}} b\left(m_{r}, m_{s}\right)=\frac{1}{\left(Q_{r+s}\right)_{r+1, s-1}} b\left(m_{r+1}, m_{s-1}\right)
$$

so that such expressions with constant sum $r+s$ are all equal to the case $r=1$, for arbitrary $s$. Using equation (2.11) twice, we are left with proving

$$
\frac{1}{\left(Q_{r+s}\right)_{r, s}}\left(Q_{s}\right)_{s-1,1} b\left(m_{r}, b\left(m_{s-1}, m_{1}\right)\right)=\frac{1}{\left(Q_{r+s}\right)_{r+1, s-1}\left(Q_{r+1}\right)_{i, 1}} b\left(m_{s-1}, b\left(m_{i}, m_{1}\right)\right) .
$$

We check the equality of each coordinate. Again by bilinearity, it suffices to prove the result by replacing the vectors $m_{r}$ and $m_{s-1}$ for canonical vectors $e_{R}$ and $e_{S-1}$, for indices corresponding to the nontrivial entries of $m_{r}$ and $m_{s-1}$ (recall the ranges of $i$ and $j$ in the induction hypothesis),

$$
k+1 \geq R \geq r, \quad S-1 \geq s-1 \geq 1 .
$$

We must prove

$$
\begin{aligned}
& \frac{1}{\left(Q_{r+s}\right)_{r, s}\left(Q_{j}\right)_{s-1,1}}\left\langle Q_{j} e_{R},\left(\left\langle Q_{\ell} e_{S-1}, m_{1}\right\rangle\right)_{\ell}\right\rangle \\
= & \frac{1}{\left(Q_{r+s}\right)_{r+1, s-1}\left(Q_{r+1}\right)_{i, 1}}\left\langle Q_{j} e_{S-1},\left(\left\langle Q_{\ell} e_{R}, m_{1}\right\rangle\right)_{\ell}\right\rangle .
\end{aligned}
$$

The argument splits in four cases: $R<k+1$ or not, $S<k+2$ or not.

(I) $R \geq k+1, S \geq k+2$

Only the first $k$ first columns of $Q_{j}$ are nonzero. Thus $Q_{j} e_{R}=Q_{j} e_{S-1}=0$.

(II) $R \geq k+1,1<S<k+2$

Again, the left hand side is zero. We consider the right hand side. We 
have $Q_{j} e_{S-1}=c e_{k+1-S+1}$, for $c \neq 0$. But then, up to a nonzero constant, the rhs is $\left\langle Q_{k+1-S+1} e_{R}, m_{1}\right\rangle$, and $Q_{k+1-S+1} e_{R}$ is zero if $R>k+1-S+1$.

(III) $R<k+1, S \geq k+2$

This case is similar to the previous one.

(IV) $R<k+1,1<S<k+2$

Here, we have to take into account the constants. We use extensively the fact that $Q_{m} e_{i}=\left(Q_{m}\right)_{m-i, i} e_{m-i}$. Simplifying the left hand side,

$$
\begin{gathered}
\left\langle Q_{j} e_{R},\left(\left\langle Q_{\ell} e_{S-1}, m_{1}\right\rangle\right)_{\ell}\right\rangle=\left(Q_{j}\right)_{k+1-R, R}\left\langle Q_{k+1-R} e_{S-1}, m_{1}\right\rangle \\
=\left(Q_{j}\right)_{k+1-R, R}\left(Q_{k+1-R}\right)_{k+2-R-S, S-1}\left(m_{1}\right)_{k+2-R-S} .
\end{gathered}
$$

Similarly, in the right hand side,

$$
\left\langle Q_{j} e_{S-1},\left(\left\langle Q_{\ell} e_{R}, m_{1}\right\rangle\right)_{\ell}\right\rangle=\left(Q_{j}\right)_{k+2-S, S-1}\left(Q_{k+2-S}\right)_{k+2-R-S, R}\left(m_{1}\right)_{k+2-R-S} .
$$

Getting rid of the term $\left(m_{1}\right)_{k+2-R-S}$, we are left with verifying an expression in the entries of matrices $Q_{m}$. From the formulas for their entries, we are arrive at the proof of a combinatorial identity: the product

$$
\left[\left(\begin{array}{c}
k \\
k+1-R
\end{array}\right)+\left(\begin{array}{l}
k \\
R
\end{array}\right)\right]\left[\left(\begin{array}{c}
k-R \\
k+2-R-S
\end{array}\right)+\left(\begin{array}{c}
k-R \\
S-1
\end{array}\right)\right]\left[\left(\begin{array}{c}
R+S-1 \\
R+1
\end{array}\right)+\left(\begin{array}{c}
R+S-1 \\
S-1
\end{array}\right)\right]\left[\left(\begin{array}{l}
R \\
R
\end{array}\right)+\left(\begin{array}{l}
R \\
1
\end{array}\right)\right]
$$

should equal

$\left[\left(\begin{array}{c}k \\ k+2-S\end{array}\right)+\left(\begin{array}{c}k \\ S-1\end{array}\right)\right]\left[\left(\begin{array}{c}R+S-1 \\ R\end{array}\right)+\left(\begin{array}{c}R+S-1 \\ S\end{array}\right)\right]\left[\left(\begin{array}{c}k+1-S \\ k+2-R-S\end{array}\right)+\left(\begin{array}{c}k+1-S \\ R\end{array}\right)\right]\left[\left(\begin{array}{l}S-1 \\ S-1\end{array}\right)+\left(\begin{array}{c}S-1 \\ 1\end{array}\right)\right]$.

Start by changing variables $\tilde{S}=S-1$ (and then rename $S=\tilde{S}$ ), obtaining an expression which is symmetric in $R, S=1, \ldots, k$. Now use the fact that each pair of binomials is of the form below, which is easily simplified: for $m+1=a+b$,

$$
\left(\begin{array}{c}
m \\
a
\end{array}\right)+\left(\begin{array}{c}
m \\
b
\end{array}\right)=\frac{m !}{(a-1) !+(b-1) !}\left[\frac{1}{a}+\frac{1}{b}\right] .
$$

After simplifying factorials, one is left with a cumbersome expression with simple fractions, whose equality is easily verified.

We prove Proposition 2.3.5: IVP matrices exist.

Proof. Take $w$ and $\widetilde{w}$ as above, so that

$$
\begin{aligned}
& -w^{\prime \prime}+u w=-q(w), \quad w(0)=0, \quad w^{\prime}(0)=e_{1}, \\
& -\widetilde{w}^{\prime \prime}+u \widetilde{w}=-q(\widetilde{w}), \quad \widetilde{w}(0)=0, \quad \widetilde{w}^{\prime}(0)=a .
\end{aligned}
$$


Let $M$ be the IVP matrix with $m_{1}=a$. To get $\widetilde{w}=M w$, first multiply the differential equation for $w$ by $M$,

$$
M\left(-w^{\prime \prime}+u w\right)=-M q(w)
$$

Since $M q(w)=q(M w),-(M w)^{\prime \prime}+u M w=-q(M w)$. As $(M w)^{\prime}(0)=$ $M w^{\prime}(0)=M e_{1}=m_{1}=a$ then, by uniqueness of solutions of IVP's, $\widetilde{w}=M w$.

Lemma 2.3.7 Let $w_{1}, \ldots, w_{t+1}$, and $\widetilde{w}_{1}, \ldots, \widetilde{w}_{t+1}$ be solutions of the IVP (2.8). Then, for $j=1, \ldots, t+1, w_{1}, \ldots, w_{j}$ are Dirichlet functions if and only if the $\widetilde{w}_{1}(u), \ldots, \widetilde{w}_{j}(u)$ also are.

Proof. The IVP matrix relating both sets of solutions is lower triangular.

We characterize $u \in \mathcal{Z}\left(M_{k}\right)$ in terms of differential equations.

Proposition 2.3.8 For $k \geq 1, u \in \mathcal{Z}\left(M_{k}\right)$ if and only if the system

$$
\left\{\begin{aligned}
-w_{j}^{\prime \prime}+u w_{j} & =-h_{j}, \quad j=1, \ldots, k, \\
h_{1}=0, \quad h_{j} & =\sum_{i=1}^{j-1}\left(\begin{array}{c}
j-1 \\
i
\end{array}\right) w_{i} w_{j-i}, \quad j=2, \ldots, k,
\end{aligned}\right.
$$

has Dirichlet solutions $w_{j}, j=1, \ldots, k$, with $w_{1} \neq 0$.

Proof. Suppose $u \in \mathcal{Z}\left(M_{k}\right)$. From Proposition 2.3.4, we obtain the system

$$
-z_{j}(u)^{\prime \prime}+u z_{j}(u)=-r_{j}(u), \quad j=1, \ldots, k,
$$

showing that system (2.13) has the required solutions. For the converse, take Dirichlet solutions $w_{1}(u), \ldots, w_{k}(u)$ of system $(2.13)$ with $w_{1}(u) \neq 0$. We have to show that $u \in \mathcal{Z}\left(M_{k}\right)$. Clearly $u \in \mathcal{Z}\left(M_{1}\right)$.

Let $k \geq 2$ and $u \in \mathcal{Z}\left(M_{\ell}\right)$ for some $\ell=1, \ldots, k-1$. Then, as above, $-z_{i}(u)^{\prime \prime}+u z_{i}(u)=-r_{i}(u)$, for $i=1, \ldots, \ell$. Consider the slightly altered system

$$
\begin{cases}-z_{i}(u)^{\prime \prime}+u z_{i}(u) & =-r_{i}(u), \quad i=1, \ldots, \ell \\ -\widetilde{z}_{\ell+1}(u)^{\prime \prime}+u \widetilde{z}_{\ell+1}(u) & =-r_{\ell+1}(u), \quad \widetilde{z}(u)(0)=0, \quad \widetilde{z}(u)^{\prime}(0)=0 .\end{cases}
$$

From Lemma 2.3.7, as $w_{1}(u), \ldots, w_{\ell+1}(u)$ are Dirichlet functions, a solution $\widetilde{z}_{\ell+1}(u)$ of the above system (having the same potential $u$ as system (2.13)) is also a Dirichlet function, and therefore $\widetilde{I}_{\ell+1}(u)=\left\langle r_{\ell+1}(u), z_{1}(u)\right\rangle=0$. Thus $u \in \mathcal{Z}\left(M_{\ell+1}\right)$ and $u \in \mathcal{Z}\left(M_{k}\right)$ follows by induction.

The next result will simplify computations considerably later. 
Proposition 2.3.9 (i) Given Dirichlet solutions $w_{1}, \ldots, w_{k}, w_{k+1}$ of system (2.8) with $w_{1} \neq 0$, there exist Dirichlet solutions $\widetilde{w}_{1}, \ldots, \widetilde{w}_{k}, \widetilde{w}_{k+1}$, for arbitrary initial conditions $\widetilde{w}^{\prime}(1)=a$, for $a_{1} \neq 0$.

(ii) Let $w$ be a vector solution of the IVP (2.8) satisfying $w^{\prime}(0)=e_{1}$. Then there is $\widetilde{w}$ satisfying (2.8) such that, at a fixed point $x_{0}$ for which $w_{1}^{\prime}\left(x_{0}\right) \neq 0$, $\widetilde{w}^{\prime}\left(x_{0}\right)=e_{1}$.

Proof. The first item is immediate. We consider (ii). From Proposition 2.3.5, any solution $\widetilde{w}$ of $(2.8)$ with $\widetilde{w}(0)=0$ and $\widetilde{w}^{\prime}(0)=a, a_{1} \neq 0$, is of the form $\widetilde{w}=M w$ where $M$ is a constant invertible lower triangular matrix $M$, which is determined by its first column $m_{1}=a$. We search for an IVP matrix $M_{0}$ for which $e_{1}=M_{0} w^{\prime}\left(x_{0}\right)$, i.e., such that the first column of its inverse matrix $M_{0}^{-1}$ is $w^{\prime}\left(x_{0}\right)$. We show that there is a unique such matrix.

From equation (2.12) which specifies column $m_{j}$ in terms of $m_{j-1}$, one obtains entry $i+1$ of $m_{j}$ from the first $i$ entries of $m_{j-1}$. The diagonal entries of $M_{0}$ and of $M_{0}^{-1}$ are computed from the first entry of $w^{\prime}\left(x_{0}\right)$. These facts suffice to compute both $M_{0}$ and $M_{0}^{-1}$ simultaneously. Indeed, assume by induction that the top $j \times j$ blocks of $M_{0}$ and $M_{0}^{-1}$ are computed. Then the last row of the $(j+1) \times(j+1)$ block of $M_{0}$ is known from (2.12), with the exception of the entry $(j+1,1)$, which is obtained from equating entry $(j+1,1)$ of the equality $M_{0} M_{0}^{-1}=I$. Inverting, one obtains the $(j+1) \times(j+1)$ block of $M_{0}^{-1}$.

Finally set $\widetilde{w}=M_{0} w: \widetilde{w}$ solves the $\operatorname{IVP}(2.8)$ and $\widetilde{w}^{\prime}\left(x_{0}\right)=M_{0} w^{\prime}\left(x_{0}\right)=$ $M_{0} M_{0}^{-1} e_{1}=e_{1}$. 


\section{Every Singularity of $F$ is of Morin type}

In this chapter we prove the first item of Theorem 2.3.3: zero is a regular value of $M_{k}$. To obtain $D M_{k}$ at its zeros, we compute in Section 3.1 the directional derivatives of the functionals $\widetilde{I}_{j}$, the components of $M_{k}$. Linear independence of the gradients is shown in Section 3.2.

\section{1}

Computing $\operatorname{grad} \widetilde{I}_{j}(u)$ on $\mathcal{Z}\left(M_{k}\right)$ for $j \leq k$

Proposition 3.1.1 For $u \in \mathcal{Z}\left(M_{k}\right)$, there are $a_{1, k}, \ldots, a_{k, k} \in \mathbb{R}$, with $a_{k, k}=1$, such that, for every nonzero $v \in X$,

$$
\sum_{i=1}^{k} a_{i, k} \partial_{v} \widetilde{I}_{i}(u)=\left\langle r_{k+1}(u), v\right\rangle .
$$

There are $b_{1, k}, \ldots, b_{k, k} \in \mathbb{R}$, with $b_{k, k}=1$, such that for every nonzero $v \in X$,

$$
\partial_{v} \widetilde{I}_{k}(u)=\sum_{i=1}^{k} b_{i, k}\left\langle r_{i+1}(u), v\right\rangle .
$$

The proof is not by induction. We present the cases $k=1$ and $k=2$ separately, in order to present arguments employed along the computations. Proof. Taking the derivative of (2.4) at $u \in \mathcal{Z}\left(M_{k}\right)$ along $v$,

$$
\begin{aligned}
D F(u) \cdot & \partial_{v} z_{k}(u) \\
= & -v z_{k}(u)-\partial_{v} r_{k}(u) \\
& \quad+\sum_{i=0}^{k-1}\left(\begin{array}{c}
k-1 \\
i
\end{array}\right)\left[\partial_{v} \widetilde{I}_{i+1}(u) z_{k-i}(u)+\widetilde{I}_{i+1}(u) \partial_{v} z_{k-i}(u)\right] \\
= & -v z_{k}(u)-\partial_{v} r_{k}(u)+\sum_{i=0}^{k-1}\left(\begin{array}{c}
k-1 \\
i
\end{array}\right)\left[\partial_{v} \widetilde{I}_{i+1}(u)\right] z_{k-i}(u) .
\end{aligned}
$$

Taking the $L^{2}$-inner product with $\phi(u)$,

$$
\begin{aligned}
& \sum_{i=0}^{k-1}\left(\begin{array}{c}
k-1 \\
i
\end{array}\right)\left[\partial_{v} \widetilde{I}_{i+1}(u)\right]\left\langle z_{k-i}(u), \phi(u)\right\rangle \\
& =\left\langle v, z_{1}(u) z_{k}(u)\right\rangle+\left\langle\partial_{v} r_{k}(u), z_{1}(u)\right\rangle .
\end{aligned}
$$


For $k=1$ the above equation is $\partial_{v} \widetilde{I}_{1}(u)=\left\langle r_{2}(u), v\right\rangle$ : the equation (3.1) then holds with $a_{1,1}=1$. For $k \geq 2$,

$$
\begin{aligned}
\partial_{v} r_{k}(u) & =\sum_{i=1}^{k-1}\left(\begin{array}{c}
k-1 \\
i
\end{array}\right)\left[\left[\partial_{v} z_{i}(u)\right] z_{k-i}(u)+z_{i}(u)\left[\partial_{v} z_{k-i}(u)\right]\right] \\
& =\sum_{i=1}^{k-1}\left(\begin{array}{c}
k-1 \\
i
\end{array}\right)\left[\partial_{v} z_{i}(u)\right] z_{k-i}(u)+\sum_{i=1}^{k-1}\left(\begin{array}{c}
k-1 \\
i
\end{array}\right) z_{i}(u)\left[\partial_{v} z_{k-i}(u)\right] \\
& =\sum_{i=1}^{k-1}\left(\begin{array}{c}
k-1 \\
i
\end{array}\right)\left[\partial_{v} z_{i}(u)\right] z_{k-i}(u)+\sum_{i=1}^{k-1}\left(\begin{array}{c}
k-1 \\
k-i
\end{array}\right)\left[\partial_{v} z_{i}(u)\right] z_{k-i}(u) \\
& =\sum_{i=1}^{k-1}\left(\begin{array}{c}
k \\
i
\end{array}\right)\left[\partial_{v} z_{i}(u)\right] z_{k-i}(u),
\end{aligned}
$$

so that

$$
\left\langle\partial_{v} r_{k}(u), z_{1}(u)\right\rangle=\sum_{i=1}^{k-1}\left(\begin{array}{c}
k \\
i
\end{array}\right)\left\langle\partial_{v} z_{i}(u), z_{1}(u) z_{k-i}(u)\right\rangle
$$

and now equation (3.3) becomes

$$
\begin{aligned}
& \sum_{i=0}^{k-1}\left(\begin{array}{c}
k-1 \\
i
\end{array}\right) {\left[\partial_{v} \widetilde{I}_{i+1}(u)\right]\left\langle z_{k-i}(u), \phi(u)\right\rangle } \\
&=\left\langle v, z_{1}(u) z_{k}(u)\right\rangle+\sum_{i=1}^{k-1}\left(\begin{array}{c}
k \\
i
\end{array}\right)\left\langle\partial_{v} z_{i}(u), z_{1}(u) z_{k-i}(u)\right\rangle .
\end{aligned}
$$

Moreover, for $k=2$,

$$
\begin{aligned}
& {\left[\partial_{v} \widetilde{I}_{1}(u)\right]\left\langle z_{2}(u), z_{1}(u)\right\rangle+\left[\partial_{v} \widetilde{I}_{2}(u)\right]\langle\phi(u), \phi(u)\rangle} \\
& \quad=\left\langle v, z_{1}(u) z_{2}(u)\right\rangle+2\left\langle\partial_{v} z_{1}(u), z_{1}(u) z_{1}(u)\right\rangle \\
& \quad=\left\langle v, z_{1}(u) z_{2}(u)\right\rangle+2\left\langle\partial_{v} \phi(u), r_{2}(u)\right\rangle \\
& \quad=\left\langle v, z_{1}(u) z_{2}(u)\right\rangle+2\left\langle\partial_{v} \phi(u),-D F(u) \cdot z_{2}(u)\right\rangle \\
& \quad=\left\langle v, z_{1}(u) z_{2}(u)\right\rangle+2\left\langle-D F(u) \cdot \partial_{v} \phi(u), z_{2}(u)\right\rangle \\
& \quad=\left\langle v, z_{1}(u) z_{2}(u)\right\rangle+2\left\langle v \phi(u)-\partial_{v} \widetilde{I}_{1}(u) \phi(u), z_{2}(u)\right\rangle \\
& \quad=\left\langle v, z_{1}(u) z_{2}(u)\right\rangle+2\left\langle v z_{1}(u), z_{2}(u)\right\rangle \quad\left(\operatorname{as}\left\langle\phi(u), z_{2}(u)\right\rangle=0\right) \\
& \quad=\left\langle v, 3 z_{1}(u) z_{2}(u)\right\rangle \\
& \quad=\left\langle v, r_{3}(u)\right\rangle .
\end{aligned}
$$

Thus (3.1) holds with $a_{1,2}=\left\langle z_{2}(u), z_{1}(u)\right\rangle$ and $a_{2,2}=\langle\phi(u), \phi(u)\rangle=1$.

Let $k \geq 3$. We drop the dependence on $u$. We first decrease the largest index $k$ of the expected answer: 


$$
\begin{aligned}
\left\langle v, r_{k+1}\right\rangle= & \left\langle v, \sum_{i=1}^{k}\left(\begin{array}{c}
k \\
i
\end{array}\right) z_{i} z_{k+1-i}\right\rangle=\sum_{i=1}^{k}\left(\begin{array}{c}
k \\
i
\end{array}\right)\left\langle v z_{i}, z_{k+1-i}\right\rangle \\
= & k\left\langle v z_{1}, z_{k}\right\rangle+\left\langle v z_{k}, z_{1}\right\rangle+\sum_{i=2}^{k-1}\left(\begin{array}{c}
k \\
i
\end{array}\right)\left\langle v z_{i}, z_{k+1-i}\right\rangle \\
= & (k+1)\left\langle v, z_{1} z_{k}\right\rangle \\
& +\sum_{i=2}^{k-1}\left(\begin{array}{c}
k \\
i
\end{array}\right)\left\langle-D F \cdot \partial_{v} z_{i}-\partial_{v} r_{i}+\sum_{j=0}^{i-1}\left(\begin{array}{c}
i-1 \\
j
\end{array}\right)\left[\partial_{v} \widetilde{I}_{j+1}\right] z_{i-j}, z_{k+1-i}\right\rangle \\
= & (k+1)\left\langle v, z_{1} z_{k}\right\rangle+\sum_{i=2}^{k-1}\left(\begin{array}{c}
k \\
i
\end{array}\right)\left\langle-D F \cdot \partial_{v} z_{i}, z_{k+1-i}\right\rangle \\
& -\sum_{i=2}^{k-1}\left(\begin{array}{c}
k \\
i
\end{array}\right)\left\langle\partial_{v} r_{i}, z_{k+1-i}\right\rangle+\sum_{i=2}^{k-1}\left(\begin{array}{c}
k \\
i
\end{array}\right) \sum_{j=0}^{i-1}\left(\begin{array}{c}
i-1 \\
j
\end{array}\right)\left[\partial_{v} \widetilde{I}_{j+1}\right]\left\langle z_{i-j}, z_{k+1-i}\right\rangle .
\end{aligned}
$$

Rearranging terms,

$$
\begin{aligned}
\left\langle v, r_{k+1}\right\rangle & -\sum_{i=2}^{k-1}\left(\begin{array}{c}
k \\
i
\end{array}\right) \sum_{j=0}^{i-1}\left(\begin{array}{c}
i-1 \\
j
\end{array}\right)\left[\partial_{v} \widetilde{I}_{j+1}\right]\left\langle z_{i-j}, z_{k+1-i}\right\rangle \\
= & (k+1)\left\langle v, z_{1} z_{k}\right\rangle+\sum_{i=2}^{k-1}\left(\begin{array}{c}
k \\
i
\end{array}\right)\left\langle-D F \cdot \partial_{v} z_{i}, z_{k+1-i}\right\rangle \\
& -\sum_{i=2}^{k-1}\left(\begin{array}{c}
k \\
i
\end{array}\right)\left\langle\partial_{v} r_{i}, z_{k+1-i}\right\rangle \\
= & (k+1)\left\langle v, z_{1} z_{k}\right\rangle+\sum_{i=2}^{k-1}\left(\begin{array}{c}
k \\
i
\end{array}\right)\left\langle\partial_{v} z_{i},-D F \cdot z_{k+1-i}\right\rangle \\
& -\sum_{i=2}^{k-1}\left(\begin{array}{c}
k \\
i
\end{array}\right)\left\langle\partial_{v} r_{i}, z_{k+1-i}\right\rangle \\
= & (k+1)\left\langle v, z_{1} z_{k}\right\rangle+\sum_{i=2}^{k-1}\left(\begin{array}{c}
k \\
i
\end{array}\right)\left\langle\partial_{v} z_{i}, r_{k+1-i}\right\rangle \\
& -\sum_{i=2}^{k-1}\left(\begin{array}{c}
k \\
i
\end{array}\right)\left\langle\partial_{v} r_{i}, z_{k+1-i}\right\rangle \\
= & (k+1)\left\langle v, z_{1} z_{k}\right\rangle+\sum_{i=2}^{k-1}\left(\begin{array}{c}
k \\
i
\end{array}\right)\left\langle\partial_{v} z_{i}, \sum_{l=1}^{k-i}\left(\begin{array}{c}
k-i \\
l
\end{array}\right) z_{l} z_{k+1-i-l}\right\rangle \\
& -\sum_{i=2}^{k-1}\left(\begin{array}{c}
k \\
i
\end{array}\right)\left\langle\sum_{l=1}^{i-1}\left(\begin{array}{l}
i \\
l
\end{array}\right)\left(\partial_{v} z_{l}\right) z_{i-l}, z_{k+1-i}\right\rangle \\
= & (k+1)\left\langle v, z_{1} z_{k}\right\rangle+\sum_{i=2}^{k-1} \sum_{l=1}^{k-i}\left(\begin{array}{c}
k \\
i
\end{array}\right)\left(\begin{array}{c}
k-i \\
l
\end{array}\right)\left\langle\partial_{v} z_{i}, z_{l} z_{k+1-i-l}\right\rangle \\
& -\sum_{i=1}^{k-1} i\left(\begin{array}{c}
k \\
i
\end{array}\right)\left(\begin{array}{l}
i \\
l
\end{array}\right)\left\langle\partial_{v} z_{l}, z_{i-l} z_{k+1-i}\right\rangle . \\
& \\
&
\end{aligned}
$$

Now use $\sum_{i=2}^{k-1} \sum_{l=1}^{i-1}=\sum_{l=1}^{k-2} \sum_{i=l+1}^{k-1}$ and $\left(\begin{array}{l}k \\ i\end{array}\right)\left(\begin{array}{l}i \\ l\end{array}\right)=\left(\begin{array}{l}k \\ l\end{array}\right)\left(\begin{array}{l}k-l \\ k-i\end{array}\right)$ : 


$$
\begin{aligned}
& \left\langle v, r_{k+1}\right\rangle-\sum_{i=2}^{k-1}\left(\begin{array}{c}
k \\
i
\end{array}\right) \sum_{j=0}^{i-1}\left(\begin{array}{c}
i-1 \\
j
\end{array}\right)\left[\partial_{v} I_{j+1}\right]\left\langle z_{i-j}, z_{k+1-i}\right\rangle \\
& =(k+1)\left\langle v, z_{1} z_{k}\right\rangle \\
& +\sum_{i=2}^{k-1} \sum_{l=1}^{k-i}\left(\begin{array}{c}
k \\
i
\end{array}\right)\left(\begin{array}{c}
k-i \\
l
\end{array}\right)\left\langle\partial_{v} z_{i}, z_{l} z_{k+1-i-l}\right\rangle \\
& -\sum_{l=1}^{k-2} \sum_{i=l+1}^{k-1}\left(\begin{array}{l}
k \\
l
\end{array}\right)\left(\begin{array}{l}
k-l \\
k-i
\end{array}\right)\left\langle\partial_{v} z_{l}, z_{i-l} z_{k+1-i}\right\rangle \\
& =(k+1)\left\langle v, z_{1} z_{k}\right\rangle \\
& +\sum_{i=2}^{k-1} \sum_{l=1}^{k-i}\left(\begin{array}{c}
k \\
i
\end{array}\right)\left(\begin{array}{c}
k-i \\
l
\end{array}\right)\left\langle\partial_{v} z_{i}, z_{l} z_{k+1-i-l}\right\rangle \\
& -\sum_{i=1}^{k-2} \sum_{l=i+1}^{k-1}\left(\begin{array}{c}
k \\
i
\end{array}\right)\left(\begin{array}{l}
k-i \\
k-l
\end{array}\right)\left\langle\partial_{v} z_{i}, z_{l-i} z_{k+1-l}\right\rangle \\
& =(k+1)\left\langle v, z_{1} z_{k}\right\rangle \\
& +k\left\langle\partial_{v} z_{k-1}, z_{1} z_{1}\right\rangle \\
& +\sum_{i=2}^{k-2} \sum_{l=1}^{k-i}\left(\begin{array}{c}
k \\
i
\end{array}\right)\left(\begin{array}{c}
k-i \\
l
\end{array}\right)\left\langle\partial_{v} z_{i}, z_{l} z_{k+1-i-l}\right\rangle \\
& -k \sum_{l=2}^{k-1}\left(\begin{array}{c}
k-1 \\
k-l
\end{array}\right)\left\langle\partial_{v} z_{1}, z_{l-1} z_{k+1-l}\right\rangle \\
& -\sum_{i=2}^{k-2} \sum_{l=i+1}^{k-1}\left(\begin{array}{c}
k \\
i
\end{array}\right)\left(\begin{array}{c}
k-i \\
k-l
\end{array}\right)\left\langle\partial_{v} z_{i}, z_{l-i} z_{k+1-l}\right\rangle \\
& =(k+1)\left\langle v, z_{1} z_{k}\right\rangle \\
& +k\left\langle\partial_{v} z_{k-1}, z_{1} z_{1}\right\rangle \\
& +\sum_{i=2}^{k-2} \sum_{l=1}^{k-i}\left(\begin{array}{c}
k \\
i
\end{array}\right)\left(\begin{array}{c}
k-i \\
l
\end{array}\right)\left\langle\partial_{v} z_{i}, z_{l} z_{k+1-i-l}\right\rangle \\
& -k \sum_{l=2}^{k-1}\left(\begin{array}{c}
k-1 \\
k-l
\end{array}\right)\left\langle\partial_{v} z_{1}, z_{l-1} z_{k+1-l}\right\rangle \\
& -\sum_{i=2}^{k-2} \sum_{l=1}^{k-i-1}\left(\begin{array}{c}
k \\
i
\end{array}\right)\left(\begin{array}{c}
k-i \\
k-l-i
\end{array}\right)\left\langle\partial_{v} z_{i}, z_{l} z_{k+1-l-i}\right\rangle \\
& =(k+1)\left\langle v, z_{1} z_{k}\right\rangle \\
& +k\left\langle\partial_{v} z_{k-1}, z_{1} z_{1}\right\rangle-k\left\langle\partial_{v} z_{1}, \sum_{l=2}^{k-1}\left(\begin{array}{c}
k-1 \\
k-l
\end{array}\right) z_{l-1} z_{k+1-l}\right\rangle \\
& +\sum_{i=2}^{k-2}\left(\begin{array}{c}
k \\
i
\end{array}\right)\left\langle\partial_{v} z_{i}, \sum_{l=1}^{k-i}\left(\begin{array}{c}
k-i \\
l
\end{array}\right) z_{l} z_{k+1-l-i}\right\rangle \\
& -\sum_{i=2}^{k-2}\left(\begin{array}{c}
k \\
i
\end{array}\right)\left\langle\partial_{v} z_{i}, \sum_{l=1}^{k-i-1}\left(\begin{array}{c}
k-i \\
k-i-l
\end{array}\right) z_{l} z_{k+1-l-i}\right\rangle .
\end{aligned}
$$




$$
\begin{aligned}
& \operatorname{As}\left(\begin{array}{c}
k-i \\
k-i-l
\end{array}\right)=\left(\begin{array}{c}
k-i \\
l
\end{array}\right) \\
& \left\langle v, r_{k+1}\right\rangle-\sum_{i=2}^{k-1}\left(\begin{array}{c}
k \\
i
\end{array}\right) \sum_{j=0}^{i-1}\left(\begin{array}{c}
i-1 \\
j
\end{array}\right)\left[\partial_{v} \widetilde{I}_{j+1}\right]\left\langle z_{i-j}, z_{k+1-i}\right\rangle \\
& =(k+1)\left\langle v, z_{1} z_{k}\right\rangle+k\left\langle\partial_{v} z_{k-1}, z_{1} z_{1}\right\rangle \\
& -k\left\langle\partial_{v} z_{1}, \sum_{l=2}^{k-1}\left(\begin{array}{c}
k-1 \\
k-l
\end{array}\right) z_{l-1} z_{k+1-l}\right\rangle+\sum_{i=2}^{k-2}\left(\begin{array}{c}
k \\
i
\end{array}\right)\left\langle\partial_{v} z_{i}, \sum_{l=1}^{k-i}\left(\begin{array}{c}
k-i \\
l
\end{array}\right) z_{l} z_{k+1-l-i}\right\rangle \\
& -\sum_{i=2}^{k-2}\left(\begin{array}{c}
k \\
i
\end{array}\right)\left\langle\partial_{v} z_{i}, \sum_{l=1}^{k-i-1}\left(\begin{array}{c}
k-i \\
l
\end{array}\right) z_{l} z_{k+1-l-i}\right\rangle \\
& =(k+1)\left\langle v, z_{1} z_{k}\right\rangle+k\left\langle\partial_{v} z_{k-1}, z_{1} z_{1}\right\rangle-k\left\langle\partial_{v} z_{1}, \sum_{l=2}^{k-1}\left(\begin{array}{c}
k-1 \\
k-l
\end{array}\right) z_{l-1} z_{k+1-l}\right\rangle \\
& +\sum_{i=2}^{k-2}\left(\begin{array}{c}
k \\
i
\end{array}\right)\left\langle\partial_{v} z_{i}, z_{k-i} z_{1}\right\rangle \\
& =(k+1)\left\langle v, z_{1} z_{k}\right\rangle+\sum_{i=2}^{k-1}\left(\begin{array}{c}
k \\
i
\end{array}\right)\left\langle\partial_{v} z_{i}, z_{k-i} z_{1}\right\rangle-k\left\langle\partial_{v} z_{1}, \sum_{l=1}^{k-1}\left(\begin{array}{c}
k-1 \\
k-1-l
\end{array}\right) z_{l} z_{k-l}\right\rangle \\
& =(k+1)\left\langle v, z_{1} z_{k}\right\rangle+\sum_{i=2}^{k-1}\left(\begin{array}{c}
k \\
i
\end{array}\right)\left\langle\partial_{v} z_{i}, z_{k-i} z_{1}\right\rangle-k\left\langle\partial_{v} z_{1}, \sum_{l=1}^{k-1}\left(\begin{array}{c}
k-1 \\
l
\end{array}\right) z_{l} z_{k-l}\right\rangle \\
& =(k+1)\left\langle v, z_{1} z_{k}\right\rangle+\sum_{i=2}^{k-1}\left(\begin{array}{l}
k \\
i
\end{array}\right)\left\langle\partial_{v} z_{i}, z_{1} z_{k-i}\right\rangle \\
& -k\left\langle\partial_{v} z_{1},-z_{1} z_{k-1}+\sum_{l=1}^{k-1}\left(\begin{array}{c}
k-1 \\
l
\end{array}\right) z_{l} z_{k-l}\right\rangle \\
& =(k+1)\left\langle v, z_{1} z_{k}\right\rangle+\sum_{i=2}^{k-1}\left(\begin{array}{c}
k \\
i
\end{array}\right)\left\langle\partial_{v} z_{i}, z_{1} z_{k-i}\right\rangle \\
& +k\left\langle\partial_{v} z_{1}, z_{1} z_{k-1}\right\rangle-k\left\langle\partial_{v} z_{1}, \sum_{l=1}^{k-1}\left(\begin{array}{c}
k-1 \\
l
\end{array}\right) z_{l} z_{k-l}\right\rangle \\
& =(k+1)\left\langle v, z_{1} z_{k}\right\rangle+\sum_{i=1}^{k-1}\left(\begin{array}{c}
k \\
i
\end{array}\right)\left\langle\partial_{v} z_{i}, z_{1} z_{k-i}\right\rangle-k\left\langle\partial_{v} z_{1}, \sum_{l=1}^{k-1}\left(\begin{array}{c}
k-1 \\
l
\end{array}\right) z_{l} z_{k-l}\right\rangle \\
& =(k+1)\left\langle v, z_{1} z_{k}\right\rangle+\sum_{i=1}^{k-1}\left(\begin{array}{c}
k \\
i
\end{array}\right)\left\langle\partial_{v} z_{i}, z_{1} z_{k-i}\right\rangle-k\left\langle\partial_{v} z_{1}, r_{k}\right\rangle \\
& =(k+1)\left\langle v, z_{1} z_{k}\right\rangle+\sum_{i=1}^{k-1}\left(\begin{array}{c}
k \\
i
\end{array}\right)\left\langle\partial_{v} z_{i}, z_{1} z_{k-i}\right\rangle-k\left\langle\partial_{v} \phi,-D F \cdot z_{k}+I_{k} \phi\right\rangle \\
& =(k+1)\left\langle v, z_{1} z_{k}\right\rangle+\sum_{i=1}^{k-1}\left(\begin{array}{c}
k \\
i
\end{array}\right)\left\langle\partial_{v} z_{i}, z_{1} z_{k-i}\right\rangle-k\left\langle\partial_{v} z_{1},-D F \cdot z_{k}\right\rangle \\
& =(k+1)\left\langle v, z_{1} z_{k}\right\rangle+\sum_{i=1}^{k-1}\left(\begin{array}{c}
k \\
i
\end{array}\right)\left\langle\partial_{v} z_{i}, z_{1} z_{k-i}\right\rangle-k\left\langle-D F \cdot \partial_{v} z_{1}, z_{k}\right\rangle \\
& =(k+1)\left\langle v, z_{1} z_{k}\right\rangle+\sum_{i=1}^{k-1}\left(\begin{array}{c}
k \\
i
\end{array}\right)\left\langle\partial_{v} z_{i}, z_{1} z_{k-i}\right\rangle-k\left\langle v z_{1}, z_{k}\right\rangle \\
& =\left\langle v, z_{1} z_{k}\right\rangle+\sum_{i=1}^{k-1}\left(\begin{array}{c}
k \\
i
\end{array}\right)\left\langle\partial_{v} z_{i}, z_{1} z_{k-i}\right\rangle .
\end{aligned}
$$


By (3.4),

$$
\begin{aligned}
\sum_{i=0}^{k-1}\left(\begin{array}{c}
k-1 \\
i
\end{array}\right) & {\left[\partial_{v} \widetilde{I}_{i+1}\right]\left\langle z_{k-i}, \phi\right\rangle } \\
= & \left\langle v, r_{k+1}\right\rangle-\sum_{i=2}^{k-1} \sum_{j=0}^{i-1}\left(\begin{array}{c}
k \\
i
\end{array}\right)\left(\begin{array}{c}
i-1 \\
j
\end{array}\right)\left[\partial_{v} \widetilde{I}_{j+1}\right]\left\langle z_{i-j}, z_{k+1-i}\right\rangle
\end{aligned}
$$

So, equation (3.1) holds and $a_{k, k}=1$.

To prove the last statement, consider matrices $A=\left(a_{i, j}\right)$ and $B=\left(b_{i, j}\right)$. Clearly $B=A^{-1}$. Since $A$ is triangular with diagonal entries equal to 1 , the same is true of $B$.

The directional derivatives computed above employ the $L^{2}$-inner product. Equivalent formulae for the inner product of the ambient space $X$ are obtained by pulling back the inner product in $Y$ with the isomorphism $-\frac{d^{2}}{d x^{2}}: X \rightarrow Y$.

\section{2}

\section{Zero is a Regular Value of $M_{k}$}

There is a well known formula for the solutions of the IVP (2.8) when $w^{\prime}(0)=e_{1}$ in terms of the right hand sides and (independent) solutions $\varphi$ and $\psi$ of the homogenous equation

$$
\left\{\begin{aligned}
-\varphi^{\prime \prime}+u \varphi=0, & \varphi(0)=0, & \varphi^{\prime}(0)=1 \\
-\psi^{\prime \prime}+u \psi=0, & \psi(0)=1, & \psi^{\prime}(0)=0 .
\end{aligned}\right.
$$

Clearly $w_{1}=\varphi$. For $j \geq 2$,

$$
w_{j}(x)=\left(\int_{0}^{x} h_{j} \psi\right) \varphi(x)-\left(\int_{0}^{x} h_{j} \varphi\right) \psi(x)
$$

In Proposition 3.2.2 we prove the linear independence of the set $\left\{h_{2}, \ldots, h_{k+1}\right\}$, by considering the local behavior of the functions near $x=0$. Let $\varepsilon>0$ be small enough such that $[0, \varepsilon] \cap \varphi^{-1}(0)=\{0\}$. As $\varphi^{\prime}(0)=1$ then $\varphi(x)>0$ on $(0, \varepsilon]$. The expressions

$$
\varphi(x)=x+\int_{0}^{x}\left(\int_{0}^{s} u(t) \varphi(t) d t\right) d s, \quad \psi(x)=1+\int_{0}^{x}\left(\int_{0}^{s} u(t) \psi(t) d t\right) d s
$$

yield

$$
\varphi(x)=x+o\left(x^{2}\right) \quad \text { and } \quad \psi(x)=1+o(x) \quad \text { as } \quad x \rightarrow 0 .
$$

Lemma 3.2.1 Let $w_{j}$ and $h_{j}$ be solutions and right hand sides of system (2.8) with initial conditions $w^{\prime}(0)=e_{1}$. Then there are strictly positive numbers $\alpha_{1}, \ldots, \alpha_{k}$ and $\beta_{2}, \ldots, \beta_{k+1}$ such that, as $x \rightarrow 0$, for $j=1, \ldots, k$,

$$
w_{j}(x)=\alpha_{j} x^{3 j-2}+o\left(x^{3 j-1}\right) \quad \text { and } \quad h_{j+1}(x)=\beta_{j+1} x^{3 j-1}+o\left(x^{3 j}\right) .
$$


Proof. For $k=1$, from (3.6), as $x \rightarrow 0$,

$$
w_{1}(x)=\varphi(x)=x+o\left(x^{2}\right), \quad h_{2}(x)=\left[w_{1}(x)\right]^{2}=x^{2}+o\left(x^{3}\right) .
$$

So (3.7) holds for $k=1$ with $\alpha_{1}=\beta_{2}=1$.

Suppose the result for $p \geq 1$ : we show it for $p+1$. From the induction hypothesis, there are nonzero numbers $\alpha_{1}, \ldots, \alpha_{p}$ and $\beta_{2}, \ldots, \beta_{p+1}$ such that (3.7) holds for $j=1, \ldots, p$. As $x \rightarrow 0$,

$$
\left\{\begin{array}{l}
h_{p+1}(x) \psi(x)=\beta_{p+1} x^{3 p-1}+o\left(x^{3 p}\right), \\
h_{p+1}(x) \varphi(x)=\beta_{p+1} x^{3 p}+o\left(x^{3 p+1}\right) .
\end{array}\right.
$$

Thus

$$
\begin{gathered}
w_{p+1}(x)=\left(\int_{0}^{x} h_{p+1} \psi\right) \varphi(x)-\left(\int_{0}^{x} h_{p+1} \varphi\right) \psi(x) \\
=\left[\frac{\beta_{p+1}}{3 p} x^{3 p}+o\left(x^{3 p+1}\right)\right]\left[x+o\left(x^{2}\right)\right]-\left[\frac{\beta_{p+1}}{3 p+1} x^{3 p+1}+o\left(x^{3 p+2}\right)\right]\left[1+o\left(x^{1}\right)\right] \\
=\beta_{p+1}\left(\frac{1}{3 p}-\frac{1}{3 p+1}\right) x^{3 p+1}+o\left(x^{3 p+2}\right)=\alpha_{p+1} x^{3(p+1)-2}+o\left(x^{3(p+1)-1}\right)
\end{gathered}
$$

and

$$
\begin{aligned}
h_{p+2}(x) & =\sum_{i=1}^{p+1}\left(\begin{array}{c}
p+1 \\
i
\end{array}\right) w_{i}(x) w_{p+2-i}(x) \\
& =\sum_{i=1}^{p+1}\left(\begin{array}{c}
p+1 \\
i
\end{array}\right)\left[\alpha_{i} x^{3 i-2}+o\left(x^{3 i-1}\right)\right]\left[\alpha_{p+2-i} x^{3(p+2-i)-2}+o\left(x^{3(p+2-i)-1}\right)\right] \\
& =\sum_{i=1}^{p+1}\left(\begin{array}{c}
p+1 \\
i
\end{array}\right)\left[\alpha_{i} \cdot \alpha_{p+2-i} x^{3(p+1)-1}+o\left(x^{3(p+1)}\right)\right] \\
& =\beta_{p+2} x^{3(p+1)-1}+o\left(x^{3(p+1)}\right),
\end{aligned}
$$

where $\alpha_{p+1}=\beta_{p+1}\left(\frac{1}{3 p}-\frac{1}{3 p+1}\right)>0$ and $\beta_{p+2}=\sum_{i=1}^{p+1}\left(\begin{array}{c}p+1 \\ i\end{array}\right) \alpha_{i} \cdot \alpha_{p+2-i}>0$. Thus (3.7) also holds for $k=p+1$.

Proposition 3.2.2 For each $k \in \mathbb{N}$, zero is a regular value of $M_{k}: X \rightarrow \mathbb{R}^{k}$. Hence the sets $\mathcal{Z}\left(M_{j}\right)$ are nested manifolds and $\Sigma^{[k]}=\mathcal{Z}\left(M_{k}\right)$. For all $u \in X$, $u \in \mathcal{S}_{k}$ if and only if $M_{k}(u)=0$ and $M_{k+1}(u) \neq 0$.

Proof. From Proposition 3.1.1, the set $\left\{\operatorname{grad} \widetilde{I}_{1}(u), \ldots, \operatorname{grad} \widetilde{I}_{k}(u)\right\}$ is linearly independent if and only if the right hand sides $\left\{r_{2}(u), \ldots, r_{k+1}(u)\right\}$ of system (2.14) are. Since IVP matrices are invertible, this in turn is equivalent to the independence of the right hand sides $\left\{h_{2}(u), \ldots, h_{k+1}(u)\right\}$ associated with solutions $w_{j}$ satisfying

$$
w(0)=0, \quad w^{\prime}(0)=e_{1}, \quad j=1, \ldots, k+1 .
$$


We prove the linear independence of $\left\{h_{2}(u), \ldots, h_{k+1}(u)\right\}$. The case $k=1$ is clear as $h_{2}(u)=\left[w_{1}(u)\right]^{2} \neq 0$. For $k \geq 2$, let $c_{2}, c_{3}, \ldots, c_{k+1} \in \mathbb{R}$ be such that

$$
c_{2} h_{2}+c_{3} h_{3}+\cdots+c_{k+1} h_{k+1}=0 .
$$

By (3.7), as $x \rightarrow 0$,

$$
c_{2}\left[\beta_{2} x^{2}+o\left(x^{3}\right)\right]+c_{3}\left[\beta_{3} x^{5}+o\left(x^{6}\right)\right]+\cdots+c_{k+1}\left[\beta_{k+1} x^{3 k-1}+o\left(x^{3 k}\right)\right]=0,
$$

where $\beta_{2}, \beta_{3}, \ldots, \beta_{k+1}$ are positive numbers. Clearly $c_{2}=0$ otherwise $x^{2}$ is an $o\left(x^{3}\right)$-function, a contradiction. Similarly, $c_{3}=\cdots=c_{k+1}=0$.

From Proposition 2.3.2, $\Sigma^{[k]}=\mathcal{Z}\left(M_{k}\right)$. The last statement is easy.

At this point we completed the proof of the first two items of Theorem 2.3.3. We next prove item (iii). In Proposition 4.4.2 we prove (iv).

\section{3}

\section{Every critical point belongs to some $\mathcal{Z}\left(M_{k}\right) \backslash \mathcal{Z}\left(M_{k+1}\right)$}

We prove item (iii) of Theorem 2.3.3.

Proposition 3.3.1 For each $u \in X$ there is $j \in \mathbb{N}$ such that $M_{j}(u) \neq 0$.

Proof. Suppose by contradiction that there is $u_{0} \in X$ such that $M_{k}\left(u_{0}\right)=0$ for all $k \in \mathbb{N}$. Hence, for every $k \in \mathbb{N}_{0}$,

$$
\partial_{\phi\left(u_{0}\right)}^{(k)} \widetilde{I}_{1}\left(u_{0}\right)=\widetilde{I}_{k+1}\left(u_{0}\right)=0
$$

From Proposition 2.1.1, $\widetilde{I}_{1}: X \rightarrow \mathbb{R}$ is a real analytic map when restricted to the line $\left\{u_{0}+t \phi\left(u_{0}\right) \mid t \in \mathbb{R}\right\}$, and thus the function

$$
t \in \mathbb{R} \mapsto \widetilde{I}_{1}\left(u_{0}+t \phi\left(u_{0}\right)\right) \in \mathbb{R}
$$

is identically zero: the line $u_{0}+\mathbb{R} \phi\left(u_{0}\right)$ is contained in $\mathcal{C}$. For the curve of eigenfuctions $t \in \mathbb{R} \mapsto \omega(t)=\phi\left(u_{0}+t \phi\left(u_{0}\right)\right) \in X$, for $t \in \mathbb{R}$,

$$
-\omega(t)^{\prime \prime}+\left[u_{0}+t \phi\left(u_{0}\right)\right] \omega(t)=0 .
$$

Setting $\omega^{(j)}(t)=\frac{\partial^{j}}{\partial t^{j}} \omega(t)$ for $j \in \mathbb{N}_{0}$, for every $k \in \mathbb{N}$,

$$
-\omega^{(k)}(t)^{\prime \prime}+\left[u_{0}+t \phi\left(u_{0}\right)\right] \omega^{(k)}(t)=-k \phi\left(u_{0}\right) \omega^{(k-1)}(t) .
$$

Define $\omega_{1}=\omega(0)=\phi\left(u_{0}\right)$ and $\omega_{k+1}=\omega^{(k)}(0)$. Evaluating (3.8) in $t=0$,

$$
-\omega_{k+1}^{\prime \prime}+u_{0} \omega_{k+1}=-k \phi\left(u_{0}\right) \omega_{k} .
$$


As $\phi\left(u_{0}+t \phi\left(u_{0}\right)\right)=z_{1}\left(u_{0}+t \phi\left(u_{0}\right)\right)$, we have $\omega^{(k)}(0)=\partial_{\phi\left(u_{0}\right)}^{(k)} z_{1}\left(u_{0}\right)$ and

$$
\omega_{k+1}=\partial_{\phi\left(u_{0}\right)}^{(k)} z_{1}\left(u_{0}\right)=z_{k+1}\left(u_{0}\right) \quad(k \in \mathbb{N}) .
$$

By $(3.9), r_{k+1}\left(u_{0}\right)=k \phi\left(u_{0}\right) z_{k}\left(u_{0}\right)$ and setting $k=2,3 \phi\left(u_{0}\right) z_{2}\left(u_{0}\right)=$ $2 \phi\left(u_{0}\right) z_{2}\left(u_{0}\right)$. Thus $\phi\left(u_{0}\right) z_{2}\left(u_{0}\right)=0$. Since the number of zeros of $\phi\left(u_{0}\right)$ is finite, $z_{2}\left(u_{0}\right)=0$ and then $r_{2}\left(u_{0}\right)=z_{2}\left(u_{0}\right)^{\prime \prime}-u_{0} z_{2}\left(u_{0}\right)=0$. As $r_{2}\left(u_{0}\right)=$ $z_{1}\left(u_{0}\right)^{2}=\phi\left(u_{0}\right)^{2}$, we derive $\phi\left(u_{0}\right)=0$, a contradiction. 


\section{4}

\section{Constructing Singularities of Arbitrary Depth}

We prove the second part of Theorem 1.0.2: for $k \geq 1$, the operator $F$ admits a Morin singularity of depth $k$, i.e., $\mathcal{Z}\left(M_{k}\right)=\Sigma^{[k]}$ is nonempty. The process is inductive: we show how to obtain a singularity of depth $k+1$ from a singularity of depth $k$. Section 4.2 .3 considers the case $k$ odd. The subsequent sections handle $k$ even, which is harder.

The argument is indirect. From Section 2.3, a Morin singularity of depth $k$ is a zero of $M_{k}: X \rightarrow \mathbb{R}^{k}$. We first show that this map extends smoothly to $\hat{M}_{k}: \hat{Y} \rightarrow \mathbb{R}^{k}$, where $\hat{Y}=H^{-1}([0,1])=\left(H_{0}^{1}([0,1])\right)^{*}$ is the dual space of $\hat{X}=H_{0}^{1}([0,1])$ (see $\left.[24]\right)$. We construct a zero of $\hat{M}_{k+1}$ and finally a density argument provides the required singularity in $X$.

In a nutshell, the problem is discretized: we find zeros of the maps $\hat{M}_{k}$ given by sums of deltas. Notice that we do not consider extensions of $F: X \rightarrow Y$ : the space $\hat{Y}$ contains distributions and interpretation of the term $f(u)=\frac{1}{2} u^{2}$ would be required. On the other hand, as it's shown in the next section, the relevant spectral properties of the Jacobian $D F(u): X \rightarrow Y$ hold for more general potentials $u \in \hat{Y}=H^{-1}([0,1])$.

\section{1 \\ Extending $M_{k}$}

The definition of the maps $M_{k}: X \rightarrow \mathbb{R}^{k}$ required two steps. For $u \in X$, first solve sequentially the initial value problems,

$$
-w_{j}(u)^{\prime \prime}+u w_{j}(u)=-h_{j}(u), \quad w(u)(0)=0, \quad w(u)^{\prime}(0)=a_{j},
$$

where $h_{1}(u)=0, a_{1} \neq 0$ and $h_{j}(u)=\sum_{i=1}^{j-1}\left(\begin{array}{c}j-1 \\ i\end{array}\right) w_{i}(u) w_{j-i}(u)$ for $j=2, \ldots, k$. If $w_{1}(u)$ is Dirichlet then, for $j \geq 2$, set $I_{j}(u)=\left\langle h_{j}(u), \varphi(u)\right\rangle .{ }^{1}$ Here $\varphi(u)$ is the eigenfunction associated to some eigenvalue $\lambda$ of $D F(u): X \rightarrow Y$ for which $\varphi(0)=0, \varphi^{\prime}(0)=1$. We now give meaning to both steps for $u \in \hat{Y}=H^{-1}([0,1])$.

${ }^{1}$ Originally, the functionals $I_{j}$ were described in terms of a different normalization, $I_{j}=\left\langle r_{j}, z_{1}\right\rangle$. This is not relevant, since both expressions differ by a nonzero multiplicative factor, so that the common roots $\mathcal{Z}\left(M_{j}\right)$ are the same for both definitions. 
Sturm-Liouville problems with potentials in $\hat{Y}$, and the related spectral theory, have been considered recently by a number of authors $([18,19])$. We provide a sketch of the necessary arguments.

For a smooth function $u$, the IVP

$$
-w^{\prime \prime}+u w=-h, \quad w(0)=0, \quad w^{\prime}(0)=a,
$$

admits an integral counterpart

$$
w(x)=a x+\int_{0}^{x} d s \int_{0}^{s} d t[u(t) w(t)+h(t)] .
$$

For general $u \in \hat{Y}$, we have to give meaning to the expression $\int_{0}^{s} u(t) w(t) d t$. Back to the smooth context, we can write, for a primitive $U$ (so that $U^{\prime}=u$ ),

$$
\int_{0}^{s} u(t) w(t) d t=U(s) w(s)-\int_{0}^{s} U(t) w^{\prime}(t) d t
$$

where replacing $U$ by $U+$ const has no effect in the expression. In particular, we may take $U\left(L^{2}-\right)$ orthogonal to 1 .

Set $\mathcal{W}=\left\{w \in H^{1}([0,1]) \mid w(0)=0\right\}$.

Lemma 4.1.1 Let $h \in \mathcal{W}$ and $a \in \mathbb{R}$. For each $u \in \hat{Y}$ the equation

$$
w(x)=a x+\int_{0}^{x} d s\left[U(s) w(s)-\int_{0}^{s} U(t) w^{\prime}(t) d t\right]+\int_{0}^{x} d s \int_{0}^{s} h(t) d t
$$

has a unique solution $w=w(u) \in \mathcal{W}$, defining a function

$$
w: \hat{Y} \rightarrow \mathcal{W}, u \mapsto w(u)
$$

which is weakly analytic when restricted to lines of $\hat{Y}$.

Proof. We leave the details to the reader. The key point is that $u \in \hat{Y}$ is the (distributional) derivative of a function $U \in L^{2}([0,1])$ orthogonal to the constant 1 (orthogonality specifies $U$ uniquely as a smooth function of $u$ ). Then all terms in sight belong to $L^{1}$ and the standard estimates for the iteration follow.

Thus, functions $u \in \hat{Y} \mapsto w_{j}(u), h_{j+1}(u) \in \mathcal{W}$ are well defined and weakly analytic on lines of $\hat{Y}$ (recall $w_{j} \in \mathcal{W}$, in which multiplication is defined and continuous, so that $\left.h_{j} \in \mathcal{W} \subset \hat{Y}\right)$.

Let $u \in \hat{Y}$. To give sense to an eigenfunction $\varphi(u)$, instead of the usual eigenvalue equation for the Jacobians $D F(u): X \rightarrow Y, v \mapsto-v^{\prime \prime}+u v$, we 
consider

$$
(T(u)-\lambda(u)) \cdot \varphi(u)=0
$$

for nonzero $\varphi(u) \in \hat{X} \subset \hat{Y}$ and $\lambda(u) \in \mathbb{R}$, where

$$
\begin{aligned}
T(u): \hat{X} & \rightarrow \hat{Y} \\
w & \mapsto T(u) \cdot w=\langle w, \cdot\rangle_{\hat{X}}+\langle w u, \cdot\rangle .
\end{aligned}
$$

For $w \in \hat{X}, w u$ is the element in $\hat{Y}$ such that $\langle w u, v\rangle=\langle u, w v\rangle$ for all $v \in \hat{X}$.

We prove smoothness of the eigenvalue and eigenfunction maps in the larger setting $u \in \hat{Y}$ as a consequence of the following result, Proposition 16 in [11] (see also [25]) for the current situation.

Proposition 4.1.2 Let $T\left(u_{*}\right) \in \mathcal{B}(\hat{X}, \hat{Y})$ have eigenvalue $\lambda_{*}=0 \in \mathbb{R}$ and eigenvector $\varphi_{*} \in \hat{X}$, so that $\left(T\left(u_{*}\right)-\lambda_{*} I\right) \varphi_{*}=0$. Assume that $T\left(u_{*}\right)-\lambda_{*} I$ is a Fredholm operator of index zero with one dimensional kernel, and that $\varphi_{*} \notin \operatorname{Ran}\left(T\left(u_{*}\right)-\lambda_{*} I\right)$. Let $\ell \in \hat{Y}=\hat{X}^{*}$ be a linear functional for which $\ell\left(\varphi_{*}\right)=1$ and set $W=\varphi_{*}+\operatorname{ker} \ell$. Then there is an open neighborhood $V \subset \mathcal{B}$ of $T\left(u_{*}\right)$ and unique smooth maps $\lambda: V \rightarrow \mathbb{R}$ and $\varphi: V \rightarrow W$ for which $(T-\lambda(T) I) \varphi(T)=0$ and $\lambda\left(T\left(u_{*}\right)\right)=\lambda_{*}, \varphi\left(T\left(u_{*}\right)\right)=\varphi_{*}$ with the usual analytic properties when restricted to lines of $\hat{Y}$.

In the case of interest, the operators in $V$ are of the form $T(u), u \in B \subset$ $\hat{Y}$, where $B$ is an open neighborhood of $u$. The functional $\ell$ provides a linear normalization of the eigenfunction, which simplifies the argument. Clearly, different choices of a normalization of the eigenvector are innocuous.

Proof. In order to prove smoothness, it suffices to check the hypotheses of the proposition.

- $T\left(u_{*}\right) \in \mathcal{B}(\hat{X}, \hat{Y})$.

Begin by the following fact. The multiplication map

$$
\begin{aligned}
\hat{X} \times \hat{Y}=H_{0}^{1}([0,1]) \times H^{-1}([0,1]) & \rightarrow H^{-1}([0,1])=\hat{Y} \\
(w, u) & \mapsto w u
\end{aligned}
$$

is bi-linear and continuous. Bi-linearity is clear. To show continuity take $(w, u) \in \hat{X} \times \hat{Y}$. For every $v \in \hat{X}$,

$$
\begin{aligned}
|\langle w u, v\rangle| & =|\langle u, w v\rangle| \leq\|u\|_{\hat{Y}}\|w v\|_{\hat{X}}=\|u\|_{\hat{Y}}\left\|(w v)^{\prime}\right\|_{L^{2}} \\
& =\|u\|_{\hat{Y}}\left\|w v^{\prime}+w^{\prime} v\right\|_{L_{2}} \\
& \leq\|u\|_{\hat{Y}}\left(\left\|w v^{\prime}\right\|_{L_{2}}+\left\|w^{\prime} v\right\|_{L_{2}}\right) \\
& \leq\|u\|_{\hat{Y}}\left(\|w\|_{\text {sup }}\left\|v^{\prime}\right\|_{L_{2}}+\left\|w^{\prime}\right\|_{L_{2}}\|v\|_{\text {sup }}\right) \\
& \leq 2\|u\|_{\hat{Y}}\|w\|_{\hat{X}}\|v\|_{\hat{X}} .
\end{aligned}
$$


Thus

$$
\|w u\|_{\hat{Y}} \leq 2\|w\|_{\hat{X}}\|u\|_{\hat{Y}}
$$

Therefore $T\left(u_{*}\right): \hat{X} \rightarrow \hat{Y}$ is well defined, linear and bounded:

$$
\begin{aligned}
\left\|T\left(u_{*}\right) \cdot w\right\|_{\hat{Y}} & \leq\left\|\langle w, \cdot\rangle_{\hat{X}}\right\|_{\hat{Y}}+\|w u\|_{\hat{Y}} \leq\|w\|_{\hat{X}}+2\|w\|_{\hat{X}}\left\|u_{*}\right\|_{\hat{Y}} \\
& \leq\left(1+2\left\|u_{*}\right\|_{\hat{Y}}\right)\|w\|_{\hat{X}} .
\end{aligned}
$$

- $T\left(u_{*}\right)-\lambda_{*}$ is a Fredholm operator of index zero.

For fixed $u \in \hat{Y}$, the linear operator $w \in \hat{X} \mapsto w u \in \hat{Y}$ is compact with norm bounded by $2\|u\|_{\hat{Y}}$. Compactness is clear for $u \in C^{0}$, as $w u \in C^{0}$ and the inclusion $C^{0} \hookrightarrow \hat{Y}$ is compact. For $u \in \hat{Y}$, approximate by continuous functions and recall that the uniform limit of compact operators is also compact.

Since $w \in \hat{X} \mapsto\langle w, \cdot\rangle_{\hat{X}} \in \hat{Y}$ is an isomorphism and $w \in \hat{X} \mapsto w u_{*} \in \hat{Y}$ is compact, $T\left(u_{*}\right)-\lambda_{*}$ is a Fredholm operator of index zero.

- $\operatorname{dim} \operatorname{ker}\left(T\left(u_{*}\right)-\lambda_{*}\right)=1$.

The argument is standard: if there are two independent kernel elements, there is a kernel element solving the (second order) homogeneous differential equation with trivial initial conditions at 0 .

- $\varphi_{*} \notin \operatorname{Ran}\left(T\left(u_{*}\right)-\lambda_{*}\right)$.

First note that for $u \in \hat{Y}, T(u)$ is symmetric in the sense that, for every $w, v \in \hat{X},\langle T(u) \cdot w, v\rangle=\langle T(u) \cdot v, w\rangle$ :

$$
\begin{aligned}
\langle T(u) \cdot w, v\rangle & =\langle w, v\rangle_{\hat{X}}+\langle w u, v\rangle=\langle w, v\rangle_{\hat{X}}+\langle u, w v\rangle \\
& =\langle v, w\rangle_{\hat{X}}+\langle u, v w\rangle=\langle v, w\rangle_{\hat{X}}+\langle v u, w\rangle=\langle T(u) \cdot v, w\rangle .
\end{aligned}
$$

Thus, if $w \in \hat{X}$ is such that $\left(T\left(u_{*}\right)-\lambda_{*}\right) \cdot w=\varphi_{*}$ then

$$
0 \neq\left\langle\varphi_{*}, \varphi_{*}\right\rangle=\left\langle\left(T\left(u_{*}\right)-\lambda_{*}\right) \cdot w, \varphi_{*}\right\rangle=\left\langle\left(T\left(u_{*}\right)-\lambda_{*}\right) \cdot \varphi_{*}, w\right\rangle=0 .
$$

The proposition then applies, and smoothness of eigenvalue and eigenvector maps is proved.

We finally extend $M_{k}: X \rightarrow \mathbb{R}^{k}$.

Corollary 4.1.3 Let $k \in \mathbb{N}$. For $u_{*} \in \hat{Y}$ for which $\lambda_{n}\left(u_{*}\right)=0$, consider $B \subset \hat{Y}$ defined in Proposition 4.1.2. For $j=1, \ldots, k$, the map

$$
\hat{I}_{j}: B \subset \hat{Y} \rightarrow \mathbb{R}, \quad u \mapsto\left\langle r_{j}(u), \varphi(u)\right\rangle
$$


is smooth. $\hat{M}_{k}=\left(\hat{I}_{1}, \ldots, \hat{I}_{k}\right): B \subset \hat{Y} \rightarrow \mathbb{R}^{k}$ is well defined and each coordinate is analytic on lines of $\hat{Y} \cap B$.

Proof. Let $u \in \hat{Y}$. From the lemma above, for arbitrary choices of initial conditions $a_{j}$, we can solve the recursive integral system, obtaining functions $w_{j}(u), r_{j}(u) \in \hat{X}$.

We introduce the subspace $X^{\delta} \subset \hat{Y}$ :

$$
X^{\delta}=\left\{u \in \hat{Y} \mid u=\sum_{i=1}^{N} c_{i} \delta_{x_{i}}, N \in \mathbb{N}, c_{i} \in \mathbb{R}, 0<x_{1}<\cdots<x_{N}<1\right\} \text {. }
$$

For $u \in H^{1}+X^{\delta}$, the differential equations in the definition of $\mathcal{Z}\left(\hat{M}_{k}\right)$ are easily interpreted without referring to their weak versions. More precisely, for $u=\widetilde{u}+\sum_{i=1}^{N} c_{i} \delta_{x_{i}}$,

$$
-w^{\prime \prime}+u w=-h
$$

is a standard differential equation in $(0,1) \backslash\left\{x_{1}, \ldots, x_{N}\right\}$ and, at $x_{i}$,

$$
w^{\prime}\left(x_{i}^{+}\right)-w^{\prime}\left(x_{i}^{-}\right)=c_{i} w\left(x_{i}\right)
$$

Elements of $X^{\delta}$ contain deltas, but we still call them functions, to distinguish them from possibly more complicated distributions in $\hat{Y}$.

Proposition 4.1.4 The Jacobians of $\hat{M}_{k}$ satisfy the same formula than those of $M_{k}$. For functions $u \in X^{\delta} \cap \mathcal{Z}\left(\hat{M}_{k}\right)$, zero is a regular value and, near such points, $\mathcal{Z}\left(\hat{M}_{k}\right)$ are manifolds.

Proof. The formulae for the directional derivatives of $I_{j}$ extend by smoothness of $\hat{M}_{k}$. Transversality (Proposition 3.2) has been proved by verifying properties of functions near zero: they are unaltered for $u \in X^{\delta}$.

We will construct elements of $\mathcal{Z}\left(\hat{M}_{k+1}\right)$ in $X^{\delta}$. The space $\hat{Y}$ is required because the density argument obtaining a deep singularity $u \in \mathcal{Z}\left(M_{k+1}\right)=$ $\Sigma^{[k+1]} \subset X$ cannot start from a space which is not complete, as $X^{\delta}$.

\section{2}

\section{Operating with singularities}

We introduce some techniques to generate singularities. 


\subsection{1}

\section{Scaling}

The McKean-Scovel operator acts on functions defined in $[0,1]$. We consider the obvious variation: for $I=[a, b], X_{I}=H^{2}(I) \cap H_{0}^{1}(I)$ and $Y_{I}=L^{2}(I), F_{I}: X_{I} \rightarrow Y_{I}, u \mapsto-u^{\prime \prime}+\frac{1}{2} u^{2}$. Statements about $F$ convert easily to $F_{I}$ : the critical set $\mathcal{C}_{I}$ of $F_{I}$ consists only of Morin singularities, which are characterized as zeros of a map $M_{k, I}: X_{I} \rightarrow \mathbb{R}^{k}, \Sigma_{I}^{[k]}=\mathcal{Z}\left(M_{k, I}\right)$. There are obvious counterparts $\hat{X}_{I}, \hat{Y}_{I}, X_{I}^{\delta}$.

Proposition 4.2.1 The natural scaling map $\hat{S}: \hat{X}_{I} \rightarrow \hat{X}$ (resp. the restricted scaling $\left.S: X_{I} \rightarrow X\right)$,

$$
u \mapsto v=\hat{S}(u), \quad v(x)=(b-a)^{2} u(a+(b-a) x) \text { for } x \in[0,1]
$$

is a diffeomorphism between $\mathcal{Z}\left(\hat{M}_{k, I}\right)$ and $\mathcal{Z}\left(\hat{M}_{k}\right)$ (resp. $\mathcal{Z}\left(M_{k, I}\right)$ and $\mathcal{Z}\left(M_{k}\right)$ ).

Proof. We prove the statement for the restricted scaling, to avoid cluttering the text with integrals. If $u \in X_{I}$ belongs to $u \in \mathcal{Z}\left(M_{k, I}\right)$ then there are functions $w_{1}(u), \ldots, w_{k}(u) \in X_{I}$, with $w_{1}(u) \neq 0$, satisfying the recursive system

$$
\left\{\begin{array}{c}
-w_{j}(u)^{\prime \prime}+u w_{j}(u)=-h_{j}(u), j=1, \ldots, k, \\
h_{1}(u)=0, h_{j}(u)=\sum_{i=1}^{j-1}\left(\begin{array}{c}
j-1 \\
i
\end{array}\right) w_{i}(u) w_{j-i}(u), j \geq 2 .
\end{array}\right.
$$

Set $v=S(u)$. We must find $w_{1}(v), \ldots, w_{k}(v) \in X$, with $w_{1}(v) \neq 0$, for which

$$
\left\{\begin{array}{c}
-w_{j}(v)^{\prime \prime}+v w_{j}(v)=-h_{j}(v), j=1, \ldots, k, \\
h_{1}(v)=0, h_{j}(v)=\sum_{i=1}^{j-1}\left(\begin{array}{c}
j-1 \\
i
\end{array}\right) w_{i}(v) w_{j-i}(v), j \geq 2 .
\end{array}\right.
$$

Define $w_{j}(v):[0,1] \rightarrow \mathbb{R}$ by

$$
w_{j}(v)(x)=\left(\frac{1}{b-a}\right)^{3 j-2} w_{j}(u)(a+(b-a) x) .
$$

If $k \geq 2$, for $j=2, \ldots, k$, a simple computation gives,

$$
\begin{aligned}
h_{j}(v)(x) & =\sum_{i=1}^{j-1}\left(\begin{array}{c}
j-1 \\
i
\end{array}\right) w_{i}(v)(x) w_{j-i}(v)(x) \\
& =\left(\frac{1}{b-a}\right)^{3 j-4} h_{j}(u)(a+(b-a) x) .
\end{aligned}
$$

So $-w_{j}(v)^{\prime \prime}+v w_{j}(v)=-h_{j}(v), j=1, \ldots, k$, and therefore $v \in \mathcal{Z}\left(M_{k}\right)$.

Similar computations apply to the inverse scaling $S^{-1}: X \rightarrow X_{I}: S$ is indeed a diffeomorphism between the sets $\mathcal{Z}\left(M_{k, I}\right)$ and $\mathcal{Z}\left(M_{k}\right)$.

Scaling will be frequently used in the sequel, without explicit mention. 


\subsection{2}

\section{Juxtaposition}

Let $u=\sum_{i=1}^{N} c_{i} \delta_{x_{i}} \in X_{[a, c]}^{\delta}$ and $v=\sum_{i=1}^{M} d_{i} \delta_{y_{i}} \in X_{[c, b]}^{\delta}$. The juxtaposition $(u v) \in X_{[a, b]}^{\delta}$ is

$$
(u v)=\sum_{i=1}^{N+M} e_{i} \delta_{z_{i}}
$$

where

$$
e_{i}, z_{i}= \begin{cases}c_{i}, x_{i} & \text { for } i=1, \ldots, N \\ d_{i-N}, y_{i-N} & \text { for } i=N+1, \ldots, N+M\end{cases}
$$

We extend accordingly. For example,

$$
w_{j}(u v)=\left\{\begin{array}{ll}
w_{j}(u) & \text { if } x \in[a, c] \\
w_{j}(v) & \text { if } x \in[c, b]
\end{array} .\right.
$$

From now on, whenever the information is obvious, we drop the reference to the underlying interval.

Proposition 4.2.2 Suppose $u \in \mathcal{Z}\left(\hat{M}_{k}\right) \cap X_{[a, c]}^{\delta}$ and $v \in \mathcal{Z}\left(\hat{M}_{k}\right) \cap X_{[c, b]}^{\delta}$.

1. $(u v)+\alpha \delta_{c} \in \mathcal{Z}\left(\hat{M}_{k}\right) \cap X_{[a, b]}^{\delta}$ for any $\alpha \in \mathbb{R}$.

2. If $(u v) \in \mathcal{Z}\left(\hat{M}_{k+1}\right) \cap X_{[a, b]}^{\delta}$ then either $u, v \in \mathcal{Z}\left(\hat{M}_{k+1}\right) \cap X^{\delta}$ or $u, v \notin$ $\mathcal{Z}\left(\hat{M}_{k+1}\right) \cap X^{\delta}$.

Proof. To prove (1), we solve the differential system (2.8) for $w_{j}(u v)$ piecewise, starting from point $c$ in both cases, as in Proposition 2.3.9. More precisely, in $[a, c]$, consider Dirichlet solutions $w_{j}(u) \in \hat{X}_{[a, c]}$ of system (2.8) with $w_{1}^{\prime}(u)(c)=1, w_{j}^{\prime}(u)(c)=0, j=2, \ldots, k$. In $[c, b]$, solve the system for Dirichlet solutions $w_{j}(v) \in \hat{X}_{[c, b]}$ satisfying $w_{j}^{\prime}(v)(c)=w_{j}^{\prime}(u)(c)$. The derivatives of $w_{j}$ are the same at both sides of $c$ and, close to $c$, both $u$ and $v$ belong to $X$ (indeed, they are zero near $c$ ). The functions $w_{j}(u v)(x)$ then solve the system in $[a, b]$ for the potential $(u v)$. Furthermore, the same functions solve the system also for the potential $(u v)+\alpha \delta_{c}$. Indeed, at $c$, both $w_{j}(u)$ and $w_{j}(v)$ are zero, and the term $\alpha \delta_{c}$ is irrelevant in the verification that $w_{j}(u v)$ solves $-w_{j}(u v)+\left((u v)+\alpha \delta_{c}\right) w_{j}(u v)=-h_{j}(u v)$.

To prove $(2)$, let $w_{1}(u v), \ldots, w_{k}(u v), w_{k+1}(u v) \in \hat{X}_{[a, b]}, w_{1}(u v) \neq 0$, solve the usual system for the potential $(u v)$. Note that the restrictions of these functions to the intervals $[a, c]$ and $[b, c]$ solve the usual system for the potentials $u$ and $v$, respectively, and since $u, v \in \mathcal{Z}\left(\hat{M}_{k}\right)$ then such restrictions 
are Dirichlet for $j=1, \ldots, k$. Now

$$
\begin{aligned}
u \in \mathcal{Z}\left(\hat{M}_{k+1}\right) & \Leftrightarrow\left(w_{k+1}(u v)\left\lceil_{[a, c]}\right)(c)=0\right. \\
& \Leftrightarrow\left(w_{k+1}(u v)\left\lceil_{[c, b]}\right)(c)=0 \Leftrightarrow v \in \mathcal{Z}\left(\hat{M}_{k+1}\right) .\right.
\end{aligned}
$$

Thus juxtaposition of two functions in $\mathcal{Z}\left(\hat{M}_{k}\right) \cap X^{\delta}$ provides a line in $\mathcal{Z}\left(\hat{M}_{k}\right) \cap X^{\delta}$. Successive juxtapositions yield additional degrees of freedom, which may be adjusted to obtain a function $z$ satisfying an additional scalar request, $z \in \mathcal{Z}\left(\hat{M}_{k+1}\right) \cap X^{\delta}$. For odd $k$, the process is much simpler: this is the content of Proposition 4.2.3.

\subsection{3}

\section{Symmetrization: from odd depth $k$ to depth $k+1$}

Let $u=\sum_{i=-N}^{-1} c_{i} \delta_{x_{i}} \in X_{[-1,0]}^{\delta}$. The symmetrization $\widehat{u} \in X_{[-1,1]}^{\delta}$ of $u$ is the juxtaposition of $u$ with its reflection $u_{r}, \widehat{u}=\left(u u_{r}\right)$ :

$$
\widehat{u}=\sum_{i=-N}^{-1} c_{i} \delta_{x_{i}}+\sum_{i=1}^{N} c_{i} \delta_{x_{i}}
$$

where, for $i=1, \ldots, N, c_{i}=c_{-i}$ and $x_{i}=-x_{-i}$.

Proposition 4.2.3 Let $k$ be odd. If $u \in \mathcal{Z}\left(\hat{M}_{k}\right)$ then $\widehat{u} \in \mathcal{Z}\left(\hat{M}_{k+1}\right)$.

Proof. From Proposition 2.3.5 and Corollary 2.3.9, for $u \in \mathcal{Z}\left(\hat{M}_{k}\right)$ the IVP starting from $x=0$ in $[-1,0]$ admits Dirichlet solutions

$$
\begin{gathered}
-w_{j}(u)^{\prime \prime}+u w_{j}(u)=-h_{j}(u), \quad j=1, \ldots, k, \\
h_{1}(u)=0, \quad h_{j}(u)=\sum_{i=1}^{j-1}\left(\begin{array}{c}
j-1 \\
i
\end{array}\right) w_{i}(u) w_{j-i}(u), \quad j=2, \ldots, k, \\
w_{1}(u)(0)=w_{j}(u)(0)=0, \quad w_{1}(u)(-1)=w_{j}(u)(-1)=0, \quad j=2, \ldots, k, \\
w_{1}(u)^{\prime}(0)=1, \quad w_{j}(u)^{\prime}(0)=0, \quad j=2, \ldots, k .
\end{gathered}
$$

A right hand side $h_{k+1}$ is obtained from the known functions $w_{1}, \ldots, w_{k}$. Using Proposition 2.3.4, we show that $\widehat{I}_{k+1}(\widehat{u})=\left\langle h_{k+1}(\hat{u}), \phi(\hat{u})\right\rangle=0$, which then implies $\widehat{u} \in \mathcal{Z}\left(\hat{M}_{k+1}\right)$.

For $j=1, \ldots, k$, define the odd extensions $w_{j}(\widehat{u}):[-1,1] \rightarrow \mathbb{R}$ by

$$
w_{j}(\widehat{u})(x)=\left\{\begin{array}{ll}
w_{j}(u)(x) & \text { if } x \in[-1,0] \\
(-1)^{j} w_{j}(u)(-x) & \text { if } x \in[0,1]
\end{array} .\right.
$$


From the formulas for $h_{j}$,

$$
h_{j}(\widehat{u})(x)=\left\{\begin{array}{ll}
h_{j}(u)(x) & \text { if } x \in[-1,0] \\
(-1)^{j} h_{j}(u)(-x) & \text { if } x \in[0,1]
\end{array} .\right.
$$

Thus, changes of sign of $w_{j}(\widehat{u})$ and $h_{j}(\widehat{u})$ are simultaneous and satisfy

$$
-w_{j}(\widehat{u})^{\prime \prime}+u w_{j}(\widehat{u})=-h_{j}(\widehat{u}), \quad j=1, \ldots, k
$$

in $[-1,1]$, as the derivatives of $w_{j}(\widehat{u})$ at both sides of zero are equal for $j=1$ (as $w_{1}$ is odd) or, even more, are equal to zero, by the prescribed initial conditions, for $j>1$. Thus, $\widehat{u} \in \hat{M}_{k} \cap X_{[-1,1]}^{\delta}$. Moreover, from the formula, $h_{k+1}(\widehat{u})$ is even. Since $w_{1}(\widehat{u})$ is an odd function, we have $\widehat{I}_{k+1}(\widehat{u})=0$, as desired.

\section{3}

\section{Some deep singularities in $X^{\delta}$}

The results in this chapter were inspired by extensive symbolic computation. Two key ingredients have been presented: (a) for $k$ odd, symmetrization allows to obtain singularities of depth $k+1$ from singularities of depth $k$, (b) juxtaposition generates a line of singularities of depth $k$, and possibly a choice of a point in such line is of depth $k+1$.

From these ingredients, by sheer computation, potentials $u_{k} \in \mathcal{Z}\left(\hat{M}_{k}\right)$ up to $k=11$ were derived out of the simplest possible eigenfunction (the case $k=1$ ). More precisely, split $I_{k}=\left[-2^{k}, 2^{k}\right]$ in $2^{k}$ equal intervals, and assign $2^{k}+1$ deltas at their endpoints (zero at the extremes $-2^{k}$ and $2^{k}$ ). We list the weights of the deltas, for depth $k$ from 1 to 5 .

$$
\begin{gathered}
(0,-2,0) \\
(0,-2,0,-2,0) \\
(0,-2,0,-2,0,-2,-106 / 21,-2,0) \\
(0,-2,0,-2,0,-2,-106 / 21,-2,0,-2,-106 / 21,-2,0,-2,0,-2,0) \\
(0,-2,0,-2,0,-2,-106 / 21,-2,0,-2,-106 / 21,-2,0,-2,0,-2,0,-2,0, \\
-2,-30971960 / 2300571,-2,-106 / 21,-2,0,-2,-106 / 21,-2,0,-2,0,-2,0)
\end{gathered}
$$

The adjusting points (which may be guessed from the values) are at entries $6,20,88,336, \ldots$ (entries are counted from 0 ). Going from $k$ odd to $k+1$ is just symmetrization, other transitions require adjustment. We do not know if the process continues indefinitely: up to $k=11$, the equation for the 
point to choose in the line of potentials in $\mathcal{Z}\left(\hat{M}_{k}\right)$ is a linear polynomial (!) with nonzero leading coefficient, and the computation of the adjusting value is trivial.

In order to complete the proof of Theorem 1.0.2, we must show that appropriate juxtapositions yield lines giving rise to such simple, solvable equations. The argument is of an existential nature.

An alternative scenario may serve as a warning. Suppose one searches for a normal form of deep Morin singularities in $\mathbb{R}^{n}$ for which all Jacobians are symmetric matrices. The same ingredients used above, starting with

$$
\left(\begin{array}{ccc}
2 & -1 & 0 \\
-1 & 2 & -1 \\
0 & -1 & 2
\end{array}\right)
$$

and the potential $u=(-2,0,-2)$ (in perfect analogy with the case $k=2$ above, as extremal coordinates do not come up in the Dirichlet discretization) gives rise to a sequence of potentials $u_{k}, k=2, \ldots, 6$, yielding in turn normal forms (in $\mathbb{R}^{2^{k}-1}$ ) of the first Morin singularities. But the computations break down for $k=7$ : the linear equation to be solved has zero leading coefficient.

Still, the proof of Theorem 1.0.2 can be adapted to show the existence of these special normal forms.

In the next section, we introduce the appropriate transversality hypothesis which guarantees that arbitrarily deep singularities may be obtained from the two basic ingredients.

\section{4}

\section{Adjustable Functions}

We consider adjustable functions. Section 4.4.1 shows how to get functions in $\mathcal{Z}\left(\hat{M}_{2 \ell+1}\right)$ from an adjustable function $\vartheta \in \mathcal{Z}\left(\hat{M}_{2 \ell}\right) \cap X^{\delta}$. Section 4.4 .2 proves the existence of such $\vartheta$.

Set $\ell \in \mathbb{N}$. A function $\vartheta \in \mathcal{Z}\left(\hat{M}_{2 \ell}\right) \cap X_{[a, b]}^{\delta}$ is adjustable at $x_{*} \in(a, b)$ if and only if there exist $u \in \mathcal{Z}\left(\hat{M}_{\ell}\right) \cap X_{\left[a, x_{*}\right]}^{\delta} \backslash \mathcal{Z}\left(\hat{M}_{\ell+1}\right)$ and $v \in \mathcal{Z}\left(\hat{M}_{\ell}\right) \cap X_{\left[x_{*}, b\right]}^{\delta} \backslash$ $\mathcal{Z}\left(\hat{M}_{\ell+1}\right)$ such that $\vartheta=(u v)$. We then write $\vartheta=(u \mid v)$. Both $u$ and $v$ have no term $c \delta_{x_{*}}$. As we will see, juxtaposition of $u$ and $v$ then yields a line in $\mathcal{Z}\left(\hat{M}_{2 \ell}\right) \cap X_{[a, b]}^{\delta}$ through $\vartheta$ containing an element $\vartheta^{*} \in \mathcal{Z}\left(\hat{M}_{2 \ell+1}\right)$. The number $x_{*}$ is called the adjustment point of $\vartheta$. 


\subsection{1}

From even $k$ to $k+1$

The whole section is dedicated to the proof of Proposition 4.4.2.

Lemma 4.4.1 Let $M$ be an IVP matrix with first column given by

$$
m_{1}=\left(1,0, \ldots, 0, m_{\ell+1,1}, \ldots, m_{2 \ell+1,1}\right) .
$$

Then

1. $m_{j, j}=1, \quad j=1, \ldots, 2 \ell+1$.

2. $m_{j, s}=0, \quad s=2, \ldots, 2 \ell, \quad j=s+1, \ldots, \min \{s+\ell-1,2 \ell+1\}$.

3. $m_{\ell+p+1, p+1}=c_{\ell, p} \cdot m_{\ell+1,1}, \quad c_{\ell, p} \neq 0, \quad p=1, \ldots, \ell$.

Recall that $M$ is a lower triangular matrix. For $\ell \geq 2$,

$$
M=\left(\begin{array}{ccccc}
1 & & & & \\
0 & \ddots & & & \\
m_{\ell+1,1} & & 1 & & \\
\vdots & \ddots & 0 & \ddots & \\
m_{2 \ell+1,1} & \cdots & m_{2 \ell+1, \ell+1} & 0 & 1
\end{array}\right)_{(2 \ell+1) \times(2 \ell+1)}
$$

Proof. From Proposition 2.3.5, for $s \geq 2$ and $j \geq s$,

$$
m_{j, s}=\frac{1}{s-1} \sum_{i=1}^{j-s+1}\left(\begin{array}{c}
j-1 \\
i
\end{array}\right) m_{i, 1} m_{j-i, s-1}
$$

Recall that $m_{i, 1}=0$ for $2 \leq i \leq \ell$.

1. Indeed, $m_{j, j}=\left(m_{1,1}\right)^{j}$.

2. For $2 \leq s \leq \ell+1$ or $\ell+2 \leq s \leq 2 \ell$ we have $2 \leq j-s+1 \leq \ell$. Then

$$
m_{j, s}=\frac{1}{s-1} \sum_{i=1}^{j-s+1}\left(\begin{array}{c}
j-1 \\
i
\end{array}\right) m_{i, 1} \cdot m_{j-i, s-1}=\frac{j-1}{s-1} m_{j-1, s-1} .
$$

Iterate: $m_{j, s}=0$, from $m_{i, 1}=0$ for $2 \leq i \leq \ell$.

3. If $p=1, \ldots, \ell$,

$$
\begin{aligned}
m_{\ell+p+1, p+1} & =\frac{1}{p} \sum_{i=1}^{\ell+1}\left(\begin{array}{c}
\ell+p \\
i
\end{array}\right) m_{i, 1} \cdot m_{\ell+p+1-i, p} \\
& =\frac{\ell+p}{p} m_{1,1} \cdot m_{\ell+p, p}+\frac{1}{p}\left(\begin{array}{c}
\ell+p \\
\ell+1
\end{array}\right) m_{\ell+1,1} \cdot m_{p, p} \\
& =\frac{\ell+p}{p} m_{\ell+p, p}+\frac{1}{p}\left(\begin{array}{l}
\ell+p \\
\ell+1
\end{array}\right) m_{\ell+1,1} .
\end{aligned}
$$


By induction, $m_{\ell+p+1, p+1}=(p+1)\left(\prod_{i=2}^{p+1} \frac{\ell+i}{i}\right) m_{\ell+1,1}=c_{\ell, p} \cdot m_{\ell+1,1}$, where $c_{\ell, p}=(p+1)\left(\prod_{i=2}^{p+1} \frac{\ell+i}{i}\right) \neq 0$ only depends of $\ell$ and $p$.

Proposition 4.4.2 If there is an adjustable function in $\mathcal{Z}\left(\hat{M}_{2 \ell}\right)$, then $\mathcal{Z}\left(\hat{M}_{2 \ell+1}\right)$ is not empty.

Proof. Set $\ell \geq 1$ and let $\vartheta \in \mathcal{Z}\left(\hat{M}_{2 \ell}\right) \cap X^{\delta}$ be an adjustable function with adjustment point $x_{*} \in(0,1)$. Let also

$$
w(\vartheta)=\left(w_{1}(\vartheta), \ldots, w_{2 \ell}(\vartheta), w_{2 \ell+1}(\vartheta)\right):[0,1] \rightarrow \mathbb{R}^{2 \ell+1}
$$

with $w_{1}(\vartheta) \neq 0$, solving

$$
-w(\vartheta)^{\prime \prime}+\vartheta w(\vartheta)=-q(w(\vartheta)), \quad w(\vartheta)(0)=0 .
$$

Since $\vartheta \in \mathcal{Z}\left(\hat{M}_{2 \ell}\right)$, by Proposition 2.3.7, the first $2 \ell$ components of $w(\vartheta)$ are Dirichlet. Note that $w_{2 \ell+1}(\vartheta)(1)$ is not necessarily zero: we only know $\vartheta \in \mathcal{Z}\left(\hat{M}_{2 \ell}\right)$. Moreover, as $\vartheta$ is adjustable and different solutions of the system (4.1) are related by (lower triangular) IVP matrices, we have

$$
w_{1}(\vartheta)\left(x_{*}\right)=\cdots=w_{\ell}(\vartheta)\left(x_{*}\right)=0 \quad \text { and } \quad w_{\ell+1}(\vartheta)(0) \neq 0 .
$$

Set $\alpha=w(\vartheta)\left(x_{*}\right)$ : we have $\alpha_{1}=\cdots=\alpha_{\ell}=0$ and $\alpha_{\ell+1} \neq 0$. Also, from Proposition 2.3.9, $w(\vartheta)$ can be chosen so that

$$
w(\vartheta)^{\prime}\left(x_{*}\right)=e_{1} \in \mathbb{R}^{2 \ell+1} .
$$

Consider the line

$$
C \in \mathbb{R} \mapsto \vartheta(C)=\vartheta+C \cdot \delta_{x_{*}} \in X^{\delta}
$$

We have that to find $w(\vartheta(C)):[0,1] \rightarrow \mathbb{R}^{2 \ell+1}$, with $w_{1} \neq 0$ and Dirichlet components $w_{j}, j=1, \ldots, 2 k$, which solves

$$
-w(\vartheta(C))^{\prime \prime}+\vartheta(C) w(\vartheta(C))=-q(w(\vartheta(C))), \quad w(\vartheta(C))(0)=0 .
$$

Clearly, the solution must be continuous and also must take into account the jump in the derivative at $x_{*}$,

$$
w(\vartheta(C))^{\prime}\left(x_{*}^{+}\right)=w(\vartheta(C))^{\prime}\left(x_{*}^{-}\right)+C \cdot w(\vartheta(C))\left(x_{*}\right) .
$$


We define $w(\vartheta(C))$ such that $w(\vartheta(C))=w(\vartheta)$ in $\left[0, x_{*}\right]$. The required jump of the derivative at $x_{*}$ is given by

$$
w(\vartheta(C))^{\prime}\left(x_{*}^{+}\right)=e_{1}+C \cdot \alpha
$$

Let $M$ be the $(2 \ell+1) \times(2 \ell+1)$ IVP matrix such that $M w(\vartheta)^{\prime}\left(x_{*}\right)=$ $e_{1}+C \cdot \alpha$. As $w(\vartheta)^{\prime}\left(x_{*}\right)=e_{1}$ then $m_{1}=M e_{1}=e_{1}+C \cdot \alpha$, i.e.,

$$
m_{1}=\left(1,0, \ldots, 0, C \cdot \alpha_{\ell+1}, \ldots, C \cdot \alpha_{2 \ell+1}\right), \quad \alpha_{\ell+1} \neq 0
$$

and, in particular, $m_{\ell+1,1}=C \cdot \alpha_{\ell+1}$. In the interval $\left[x_{*}, 1\right]$, the functions $w(\vartheta)$ and $M w(\vartheta)$ at $x_{*}$ may be different. From the form of the IVP matrix $M$ (Lemma 4.4.1) and since $m_{2 \ell+1, \ell+1}=c_{\ell, \ell} \cdot m_{\ell+1,1}, c_{\ell, \ell} \neq 0$ (item (3)),

$$
\begin{aligned}
M w(\vartheta) & \left(x_{*}\right)-w(\vartheta)\left(x_{*}\right)=M \alpha-\alpha \\
& =m_{2 \ell+1, \ell+1} \cdot \alpha_{\ell+1} e_{2 \ell+1} \\
& =c_{\ell, \ell} \cdot m_{\ell+1,1} \cdot \alpha_{\ell+1} e_{2 \ell+1} \\
& =c_{\ell, \ell} \cdot\left(\alpha_{\ell+1}\right)^{2} \cdot C e_{2 \ell+1} \\
& =A_{2 \ell+1} \cdot C e_{2 \ell+1},
\end{aligned}
$$

where $A_{2 \ell+1}=c_{\ell, \ell} \cdot\left(\alpha_{\ell+1}\right)^{2} \neq 0$ does not depend of $C$.

Now define

$$
w(\vartheta(C))=\left\{\begin{array}{ll}
w(\vartheta) & \text { if } x \in\left[0, x_{*}\right] \\
M w(\vartheta)-A_{2 \ell+1} \cdot C \cdot \psi(\vartheta) e_{2 \ell+1} & \text { if } x \in\left[x_{*}, 1\right]
\end{array},\right.
$$

where $\psi(\vartheta):\left[x_{*}, 1\right] \rightarrow \mathbb{R}$ solves

$$
-\psi(\vartheta)^{\prime \prime}+\vartheta \psi(\vartheta)=0, \quad \psi(\vartheta)\left(x_{*}\right)=1, \quad \psi(\vartheta)^{\prime}\left(x_{*}\right)=0
$$

Clearly $w(\vartheta(C))$ solves (4.2), is continuous and the jump (4.3) of the derivative is satisfied, as $\psi(\vartheta)^{\prime}\left(x_{*}\right)=0$. Moreover, the first $2 \ell$ components of $w(\vartheta(C))$ are Dirichlet (Proposition 2.3.7).

To handle the last component, we start by proving the following fact: $w_{2 \ell+1}(\vartheta(C))(1)$ is a non-constant linear polynomial in the variable $C$.

First recall that $\psi(\vartheta)$ solves $-z^{\prime \prime}+\vartheta z=0$ in $\left[x_{*}, 1\right]$, with $\psi(\vartheta)\left(x_{*}\right)=1$, $\psi(\vartheta)^{\prime}\left(x_{*}\right)=0$. Also, $w_{1}(\vartheta)$ is a Dirichet solution to the same equation. From the Wronskian identity, $\psi(\vartheta)(1) \neq 0$. Therefore $A_{2 \ell+1} \cdot \psi(\vartheta)(1) \neq 0$ and 


$$
\begin{aligned}
w_{2 \ell+1}(\vartheta(C))(1) & =\left\langle e_{2 \ell+1}, M w(\vartheta)(1)\right\rangle-A_{2 \ell+1} \cdot C \cdot \psi(\vartheta)(1) \\
& =\left\langle M e_{2 \ell+1}, w(\vartheta)(1)\right\rangle-A_{2 \ell+1} \cdot C \cdot \psi(\vartheta)(1) \\
& =\left\langle e_{2 \ell+1}, w(\vartheta)(1)\right\rangle-A_{2 \ell+1} \cdot C \cdot \psi(\vartheta)(1) . \\
& =w_{2 \ell+1}(\vartheta)(1)-A_{2 \ell+1} \cdot C \cdot \psi(\vartheta)(1) .
\end{aligned}
$$

As $w_{2 \ell+1}(\vartheta)(1)$ is a constant which does not depend of $C$, we are done.

We are ready to complete the proof of the proposition. From the fact above, we can choose $C=C^{*}$ such that $w_{2 \ell+1}\left(\vartheta\left(C^{*}\right)\right)(1)=0$ and therefore, for $\vartheta^{*}=\vartheta\left(C^{*}\right)=\vartheta+C^{*} \cdot \delta_{x_{*}}$, all the components of $w\left(\vartheta^{*}\right)$ are Dirichlet: $\vartheta^{*}$ belongs to $\mathcal{Z}\left(\hat{M}_{2 \ell+1}\right) \cap X^{\delta}$.

\subsection{2}

\section{Existence of Adjustable Functions}

In Proposition 4.4.5 we obtain adjustable functions in $\mathcal{Z}\left(\hat{M}_{2 \ell}\right) \cap X^{\delta}$ for $\ell \in \mathbb{N}$. As an example, which is also the first step in the inductive argument, we show how to obtain an adjustable function in $\mathcal{Z}\left(\hat{M}_{2}\right) \cap X^{\delta}$. In other words, we show that there are $u, v \in \mathcal{Z}\left(\hat{M}_{1}\right) \cap X^{\delta} \backslash \mathcal{Z}\left(\hat{M}_{2}\right)$ such that $(u \mid v) \in \mathcal{Z}\left(\hat{M}_{2}\right)$.

Set $u=-2 \delta_{-1} \in X_{[-2,0]}^{\delta}$ and let $w_{1}(u):[-2,0] \rightarrow \mathbb{R}$ solves the IVP

$$
-w_{1}(u)^{\prime \prime}+u w_{1}(u)=0, \quad w_{1}(u)(-2)=0, \quad w_{1}(u)^{\prime}(-2)=1 .
$$

Thus

$$
w_{1}(u)(x)=\left\{\begin{array}{ll}
2+x & \text { if } x \in[-2,-1] \\
-x & \text { if } x \in[-1,0]
\end{array} .\right.
$$

Since $w_{1}(u)$ also satisfies the Dirichlet condition, $u \in \mathcal{Z}\left(\hat{M}_{1}\right)$ and $w_{1}(u)$ is an eigenfunction associated to $\lambda=0$. Moreover, $w_{1}(u)$ does not change sign in $[-2,0]$, so that $\widehat{I}_{2}(u)=\int_{-2}^{0} w_{1}(u)^{3} \neq 0$ and therefore $u \notin \mathcal{Z}\left(\hat{M}_{2}\right)$. Let $u_{r} \in \mathcal{Z}\left(\hat{M}_{1}\right) \cap X_{[0,2]}^{\delta}$ be the reflection of $u$. Clearly $u_{r} \notin \mathcal{Z}\left(\hat{M}_{2}\right)$ and $\vartheta=\widehat{u}=\left(u \mid u_{r}\right) \in X_{[-2,2]}^{\delta}$. By symmetrization (Proposition 4.2.3), $\vartheta \in \mathcal{Z}\left(\hat{M}_{2}\right)$ and is adjustable at $x_{*}=0$.

The following result is standard.

Lemma 4.4.3 $X^{\delta}$ is dense in $\hat{Y}$.

We also employ the following density theorem $([14,12])$.

Theorem 4.4.4 Let $X$ be a Banach space, $D$ a dense subspace of $X$ and $M$ a smooth submanifold of finite codimension of $X$. Then $M \cap D$ is dense in $M$. 
If $\vartheta=(u \mid v) \in \mathcal{Z}\left(\hat{M}_{2 \ell}\right)$ is adjustable, we also write $\vartheta^{*} \in \mathcal{Z}\left(\hat{M}_{2 \ell+1}\right)$ obtained in the previous section as $\vartheta^{*}=(u \uparrow v)$.

Proposition 4.4.5 For $\ell \in \mathbb{N}$, if $\mathcal{Z}\left(M_{2 \ell}\right)$ is nonempty, there exists an adjustable function $\vartheta \in \mathcal{Z}\left(\hat{M}_{2 \ell}\right) \cap X^{\delta}$.

Proof. The proof is by induction. The case $\ell=1$ was shown above. Suppose the statement holds for $\ell=1, \ldots, p-1(p \geq 2)$. Let $\mathcal{Z}\left(M_{2 p}\right) \neq \emptyset$. We must find an adjustable function $\vartheta_{2 p} \in \mathcal{Z}\left(\hat{M}_{2 p}\right) \cap X^{\delta}$.

As the $\mathcal{Z}\left(M_{j}\right)$ 's are nested then $\mathcal{Z}\left(M_{p}\right)$ is non-empty and therefore $\mathcal{Z}\left(M_{p}\right) \backslash \mathcal{Z}\left(M_{p+1}\right) \neq \emptyset$. Let $\tilde{u} \in \mathcal{Z}\left(M_{p}\right) \backslash \mathcal{Z}\left(M_{p+1}\right)$. For concreteness, suppose that $\tilde{u}$ is defined in the interval ${ }^{2}[-1,0]$. Since $X^{\delta}$ is dense in $\hat{Y}$, by density (Theorem 4.4.4), there is $u \in \mathcal{Z}\left(\hat{M}_{p}\right) \backslash \mathcal{Z}\left(\hat{M}_{p+1}\right) \cap X^{\delta}$ near $\tilde{u}$. Let $u_{r}$ be the reflection of $u$. Then $u_{r},\left(u u_{r}\right) \in \mathcal{Z}\left(\hat{M}_{p}\right)$. We first prove that, for some $v_{1}^{*} \in X^{\delta}$, we have $\left(u v_{1}^{*}\right) \in \mathcal{Z}\left(\hat{M}_{p+1}\right) \cap X^{\delta}$.

- For odd $p,\left(u u_{r}\right) \in \mathcal{Z}\left(\hat{M}_{p+1}\right) \cap X^{\delta}$ (symmetrization, Proposition 4.2.3). Take $v_{1}^{*}=u_{r}$.

- For even $p$, we must work harder. As $p / 2 \in\{1, \ldots, p-1\}$, from the inductive hypothesis, take an adjustable $\vartheta_{p}=\left(u_{p} \mid v_{p}\right) \in \mathcal{Z}\left(\hat{M}_{p}\right) \cap X^{\delta}$ with $u_{p}, v_{p} \in \mathcal{Z}\left(\hat{M}_{\frac{p}{2}}\right) \cap X^{\delta} \backslash \mathcal{Z}\left(\hat{M}_{\frac{p}{2}+1}\right)$. Then, by the properties of juxtaposition (Proposition 4.2.2),

$$
\widetilde{u}_{p}=\left(\left(u u_{r}\right) u_{p}\right)=\left(u\left(u_{r} u_{p}\right)\right) \in \mathcal{Z}\left(\hat{M}_{\frac{p}{2}}\right) \cap X^{\delta} \backslash \mathcal{Z}\left(\hat{M}_{\frac{p}{2}+1}\right)
$$

and, as $\left(u u_{r}\right), \vartheta_{p} \in \mathcal{Z}\left(\hat{M}_{p}\right)$,

$$
\left(\left(u u_{r}\right) \vartheta_{p}\right)=\left(\left(\left(u u_{r} u_{p}\right) v_{p}\right)=\left(\widetilde{u}_{p} v_{p}\right) \in \mathcal{Z}\left(\hat{M}_{p}\right) \cap X^{\delta}\right.
$$

Thus $\left(\widetilde{u}_{p} \mid v_{p}\right) \in \mathcal{Z}\left(\hat{M}_{p}\right) \cap X^{\delta}$ is adjustable. From Proposition 4.4.2, we obtain $\left.\left(\widetilde{u}_{p} \uparrow v_{p}\right)=\left(u v u_{p} \uparrow v_{p}\right)\right) \in \mathcal{Z}\left(\hat{M}_{p+1}\right) \cap X^{\delta}$.

In both cases, for $v_{1}^{*}=u_{r} \in X^{\delta}$ or $\left(\left(v u_{p}\right) \uparrow v_{p}\right) \in X^{\delta}$, respectively, we have $\left(u v_{1}^{*}\right) \in \mathcal{Z}\left(\hat{M}_{p+1}\right) \cap X^{\delta}$.

In order to obtain the adjustable function $\vartheta_{2 p} \in \mathcal{Z}\left(M_{2 p}\right) \cap X^{\delta}$, we obtain sequentially, for $q=1, \ldots, p$, adjustable functions $\vartheta_{p+q} \in \mathcal{Z}\left(M_{p+q}\right) \cap X^{\delta}$. The starting point of the induction $q=1$ is the first step above.

Suppose by induction that, for $q=1, \ldots, p-1$, there is $v_{q}^{*} \in X^{\delta}$ such that $\left(u \mid v_{q}^{*}\right) \in \mathcal{Z}\left(\hat{M}_{p+q}\right) \cap X^{\delta}$. We follow closely the argument above.

${ }^{2}$ Still, we drop references to it: new operations will naturally change the domains of definition of the functions of interest, and we systematically invoke implicitly the invariance of singularity depth under scaling (Proposition 4.2.1). 
- If $p+q$ is odd,

$$
\left(\left(u v_{q}^{*}\right) \theta_{q}\right)=\left(u\left(v_{q}^{*} \theta_{q}\right)\right) \in \mathcal{Z}\left(\hat{M}_{p+q+1}\right) \cap X^{\delta},
$$

where $\theta_{q}=\left(u v_{q}^{*}\right)_{r}$ is the reflection of $\left(u v_{q}^{*}\right)$.

- If $p+q$ is even, take an adjustable $\vartheta_{p+q}=\left(u_{p+q} \mid v_{p+q}\right) \in \mathcal{Z}\left(\hat{M}_{p+q}\right) \cap X^{\delta}$ for $u_{p+q}, v_{p+q} \in \mathcal{Z}\left(\hat{M}_{\frac{p+q}{2}}\right) \cap X^{\delta} \backslash \mathcal{Z}\left(\hat{M}_{\frac{p+q}{2}+1}\right)$. Then

$$
\widetilde{u}_{p+q}=\left(\left(u v_{q}^{*}\right) u_{p+q}\right)=\left(u\left(v_{q}^{*} u_{p+q}\right)\right) \in \mathcal{Z}\left(\hat{M}_{\frac{p+q}{2}}\right) \cap X^{\delta} \backslash \mathcal{Z}\left(\hat{M}_{\frac{p+q}{2}+1}\right)
$$

and

$$
\left(\left(u v_{q}^{*}\right) \vartheta_{p+q}\right)=\left(\left(\left(u v_{q}^{*}\right) u_{p+q}\right) \mid v_{p+q}\right)=\left(\widetilde{u}_{p+q} \mid v_{p+q}\right) \in \mathcal{Z}\left(\hat{M}_{p+q}\right) \cap X^{\delta} .
$$

Thus $\left(\widetilde{u}_{p+q} \mid v_{p+q}\right) \in \mathcal{Z}\left(\hat{M}_{p+q}\right) \cap X^{\delta}$ is adjustable and therefore $\left(\widetilde{u}_{p+q} \uparrow v_{p+q}\right)=$ $\left(u\left(\left(v_{q}^{*} u_{p+q}\right) \uparrow v_{p+q}\right)\right) \in \mathcal{Z}\left(\hat{M}_{p+q+1}\right) \cap X^{\delta}$.

Again, for $v_{q+1}^{*}=\left(u v_{q}^{*}\right)_{r} \in X^{\delta}$ or $\left(\left(v_{q}^{*} u_{p+q}\right) \uparrow v_{p+q}\right) \in X^{\delta}$, we have $\left(u v_{q+1}^{*}\right) \in \mathcal{Z}\left(\hat{M}_{p+q+1}\right) \cap X^{\delta}$. The proof of the inductive step is complete.

By setting $q=p-1$, we obtain a function $v_{p}^{*} \in X^{\delta}$ such that $\left(u v_{p}^{*}\right) \in \mathcal{Z}\left(\hat{M}_{2 p}\right) \cap X^{\delta}$. Since $u \in \mathcal{Z}\left(\hat{M}_{p}\right) \cap X^{\delta} \backslash \mathcal{Z}\left(\hat{M}_{p+1}\right)$, by Proposition 4.2 .2 , we also have $v_{p}^{*} \in \mathcal{Z}\left(\hat{M}_{p}\right) \cap X^{\delta} \backslash \mathcal{Z}\left(\hat{M}_{p+1}\right): \vartheta_{2 p}=\left(u \mid v_{p}^{*}\right) \in \mathcal{Z}\left(\hat{M}_{2 p}\right) \cap X^{\delta}$ is adjustable.

\section{5}

\section{There exist Morin singularities of arbitrary depth}

We are ready to prove the second part of Theorem 1.0.2: $F$ admits Morin singularities of arbitrary depth, i.e., $\mathcal{Z}\left(M_{k}\right) \neq \emptyset$ for all $k \geq 1$. As $\mathcal{Z}\left(M_{1}\right) \neq \emptyset$ all we need is the inductive step below.

Proposition 4.5.1 For each $k \in \mathbb{N}, \mathcal{Z}\left(M_{k}\right) \neq \emptyset$ implies $\mathcal{Z}\left(M_{k+1}\right) \neq \emptyset$.

Proof. Let $k \in \mathbb{N}$ and suppose $\mathcal{Z}\left(M_{k}\right) \neq \emptyset$. Since $\mathcal{Z}\left(M_{k}\right) \subset X \subset \hat{Y}$, then $\mathcal{Z}\left(\hat{M}_{k}\right) \cap X^{\delta} \neq \emptyset$ by density (Theorem 4.4.4).

If $k$ is odd, $\mathcal{Z}\left(\hat{M}_{k+1}\right) \cap X^{\delta} \neq \emptyset$, by symmetrization (Proposition 4.2.3).

If $k$ is even, there exists an adjustable function in $\mathcal{Z}\left(\hat{M}_{k}\right) \cap X^{\delta}$ (Proposition 4.4.5) which in turn yields a function $u \in \mathcal{Z}\left(\hat{M}_{k+1}\right) \cap X^{\delta}$ (Proposition 4.4.1).

The density argument (Theorem 4.4.4) applies since, near $u, \mathcal{Z}\left(\hat{M}_{k+1}\right)$ is a submanifold of $\hat{Y}$ of finite codimension (Proposition 4.1.4) and $X$ is dense in $\hat{Y}: X \cap \mathcal{Z}\left(\hat{M}_{k+1}\right)=\mathcal{Z}\left(M_{k+1}\right)$ is nonempty.

The proof of Theorem 1.0.2 is complete. 


\section{6}

\section{An application of Theorem 1.0.2}

McKean and Scovel [6] proved that each $g \in Y$ has at most a finite number of pre-images in $X$ under $F$. This number can be arbitrarily large, as shown by a more general result by Ruf and Solimini [16]. Here, we show that an arbitrarily large number of solutions may be arbitrarily close to each other.

Corollary 4.6.1 Given $k \in \mathbb{N}$ and $\varepsilon>0$, there exists $g \in Y$ such that

$$
-u^{\prime \prime}+\frac{1}{2} u^{2}=g, \quad u \in X
$$

has $k+1$ solutions which are at most $\varepsilon$ apart.

Proof. Let $u_{*} \in X$ be a Morin singularity of depth $k$ and consider the normal form of $F$ near $u_{*}$, given in equation (1.1). Now choose small numbers $s_{1}, \ldots, s_{k-1}$ for which the polynomial $t^{k+1}+s_{1} t^{k-1}+s_{2} t^{k-2}+s_{k-1} t$ admits some value with $k+1$ preimages. 


\section{Bibliography}

[1] AMBROSETTI, A.; PRODI, G. On the inversion of some differentiable mappings with singularities between Banach spaces. Ann. Mat. Pura Appl., 93:231-246, 1972.

[2] MANES, A.; MICHELETTI, A. M. Un estensione della teoria variazionale classica degli autovalori per operatori ellittici del secondo ordine. Boll. Un. Mat. Ital., 7:285-301, 1973.

[3] BERGER, M. S.; PODOLAK, E. On the solutions of a nonlinear Dirichlet problem. Indiana Univ. Math. Journal, 24:837-846, 1975.

[4] BERGER, M. S.; CHURCH, P. T.; TIMOURIAN, J. G. Folds and cusps in Banach spaces, with applications to nonlinear partial differential equations. i. Indiana Univ. Math. Journal, 34:1-19, 1985.

[5] BERGER, M. S.; CHURCH, P. T.; TIMOURIAN, J. G. Folds and cusps in Banach spaces, with applications to nonlinear partial differential equations. ii. Transactions of the American Mathematical Society, 307:225244, 1988.

[6] MCKEAN, H. P.; SCOVEL, J. C. Geometry of some simple nonlinear differential operators. Annali della Scuola Normale Superiore di Pisa Classe di Scienze, 13 (2):299-346, 1985.

[7] MCKEAN, H. P. Singularities of a simple elliptic operator. J. Differential Geometry, 25:157-165, 1987.

[8] MCKEAN, H. P. Correction to: Singularities of a simple elliptic operator. J. Differential Geometry, 36:255, 1992.

[9] BALBONI, F.; DONATI, F. Singularities of Fredholm maps with onedimensional kernels, I: A complete classification. arXiv: Functional Analysis, 1:1-67, 2014.

[10] CAFAGNA, V.; DONATI, F. Un résult global de multiplicité pour un problème differéntiel non linéaire du premier ordre. C. R. Acad. Sc. Paris, 300:523-526, 1985. 
[11] CALANCHI, M.; TOMEI, C.; ZACCUR, A. Abundance of cusps and a converse to the Ambrosetti-Prodi theorem. arXiv:1508.01436v1, p. 1-26, 2015.

[12] BURGHELEA, D.; SALDANHA, N. C.; TOMEI, C. Results on infinite dimensional topology and applications to the structure of the critical set of nonlinear Sturm-Liouville operators. J. Differential Equations, 188:569-590, 2003.

[13] BUENO, H.; TOMEI, C. Critical sets of nonlinear Sturm-Liouville operators of Ambrosetti-Prodi type. Nonlinearity, 15-4:1073-1077, 2002.

[14] MALTA, I.; SALDANHA, N. C.; TOMEI, C. Morin singularities and global geometry in a class of ordinary differential operators. Topological Methods in Nonlinear Analysis, 10:137-169, 1997.

[15] RUF, B. Singularity theory and bifurcation phenomena in differential equations. In: TOPOLOGICAL NONLINEAR ANALYSIS II, p. 315-395, Boston, 1997.

[16] RUF, B.; SOLIMINI, S. On a class of superlinear Sturm-Liouville problems with arbitrarily many solutions. SIM J. Math. Anal. https://doi.org/10.1137/0517055, 17-4:761-771, 1986.

[17] MORIN, B. Formes canoniques de singularités d'une application différentiable. C.R. Acad. Sc. Paris, 260:5662-5665, 6503-6506, 1965.

[18] SAVCHUK, A.M.; SHKALIKOV, A.A. Sturm-Liouville operators with distribution potentials. Trans. Moscow Math. Soc., p. 143-192, 2003.

[19] ECKHARDT, J.; GESZTESY, F.; NICHOLS, R.; TESCHL, G. Weyl-Titchmarsh theory for sturm-liouville operators with distributional potentials. Opuscula Math. http://dx.doi.org/10.7494/OpMath.2013.33.3.467, 33-3:467-563, 2013.

[20] PÖSCHEL, J.; TRUBOWITZ, E. Inverse Spectral Theory. AP, Orlando, Florida (US), 1st edition, 1987.

[21] ARNOLD, V. I.; GORYUNOV, V. V.; LYASHKO, O. V.; VASIL'EV, V. Singularity Theory I. Springer, Berlin (Ge), 1st edition, 1998.

[22] SCHMÜDGEN, K. Unbounded Self-adjoint Operators on Hilbert Space. Springer, Berlin (Ge), 1st edition, 2012. 
[23] GOHBERG, I.; GOLDBERG, S.; KAASHOEK, M. A. Basic Classes of Linear Operators. Birkhäuser, Berlin (Ge), 1st edition, 2000.

[24] BREZIS, H. Functional Analysis, Sobolev Spaces and Partial Differential Equations. Springer-Universitext, 1st edition, 2011.

[25] ZEIDLER, E. Nonlinear Functional Analysis and its Applications, IV. Springer, New York, 1997. 